TRANSACTIONS OF THE

AMERICAN MATHEMATICAL SOCIETY

Volume 357, Number 7, Pages 2939-2973

S 0002-9947(04)03650-5

Article electronically published on December 28, 2004

\title{
TRANSLATION AND SHUFFLING OF PROJECTIVELY PRESENTABLE MODULES AND A CATEGORIFICATION OF A PARABOLIC HECKE MODULE
}

\author{
VOLODYMYR MAZORCHUK AND CATHARINA STROPPEL
}

\begin{abstract}
We investigate certain singular categories of Harish-Chandra bimodules realized as the category of $\mathfrak{p}$-presentable modules in the principal block of the Bernstein-Gelfand-Gelfand category $\mathcal{O}$. This category is equivalent to the module category of a properly stratified algebra. We describe the socles and endomorphism rings of standard objects in this category. Further, we consider translation and shuffling functors and their action on the standard modules. Finally, we study a graded version of this category; in particular, we give a graded version of the properly stratified structure, and use graded versions of translation functors to categorify a parabolic Hecke module.
\end{abstract}

\section{INTRODUCTION}

Harish-Chandra bimodules for semi-simple complex Lie algebras play an important role in representation theory. Certain categories of Harish-Chandra bimodules (the "regular" ones) are now relatively well-understood since they are equivalent to blocks of the well-known category $\mathcal{O}$ of Bernstein, Gelfand and Gelfand, [BGG]. In particular, they form highest weight categories. However, the "singular" categories of Harish-Chandra bimodules are not that well understood and are more difficult to approach. The aim of the paper is to give a better description of the structure of some singular categories of Harish-Chandra bimodules using different equivalent realizations.

The categories in question are not highest weight categories in general. Recently it was shown in KM1] that these categories can be described as module categories over properly stratified algebras (in the sense of [D] $)$. On the other hand, it is well known that these categories of Harish-Chandra bimodules can be realized as certain subcategories of the principal block of the category $\mathcal{O}$. Our first task is to understand this realization more explicitly. In Section 2 we describe projective, standard and simple objects, and the abelian structure of the category. One advantage of this realization is that we have the singular situation as a subcategory of the regular one. We tried to use very general arguments in the proofs. From our point of view this might provide an approach to transfer translation principles to a more general situation of module categories (see also the formalization of properties of translation functors and their categorical setup, recently developed in [Kh]).

To state our further results we need some notation. Let $\mathfrak{g}$ denote a semi-simple finite-dimensional complex Lie algebra with a fixed triangular decomposition. The

Received by the editors January 28, 2004.

2000 Mathematics Subject Classification. Primary 17B10; Secondary 20C08, 13E10. 
isomorphism classes of indecomposable projective objects in the principal block of the corresponding category $\mathcal{O}$ are indexed by the elements of the Weyl group $W$ of $\mathfrak{g}$. Let $\mathfrak{p}$ be a parabolic subalgebra of $\mathfrak{g}$ associated with the triangular decomposition above and $W_{\mathfrak{p}} \subset W$ the corresponding parabolic subgroup. The main object of our study is the category $\mathcal{O}_{0}^{\mathfrak{p}-\text { pres }}$. This is the full subcategory of the principal block of $\mathcal{O}$, consisting of all modules $M$, having a presentation by projective modules, whose indecomposable direct summands are indexed by the elements of the set $W(\mathfrak{p})$ of distinguished right coset representatives of maximal length in $W_{\mathfrak{p}} \backslash W$.

We reprove the fact that $\mathcal{O}_{0}^{\mathfrak{p}-\text { pres }}$ is a module category over a properly stratified algebra (Theorem 2.16). For a properly stratified algebra one has a class of socalled standard modules whose role is analogous to the role of standard (Verma) modules in a highest weight category. Our first result is that standard modules in $\mathcal{O}_{0}^{\mathfrak{p}-\text { pres }}$ behave like Verma modules under translation through the wall. That is, for a standard module $\Delta(w)$ and a simple reflection $s$ we have (Proposition 4.2) the following short exact sequences (both in the categories $\mathcal{O}_{0}$ and $\mathcal{O}_{0}^{\mathfrak{p}-\text { pres }}$ ):

$$
\begin{array}{llllll}
\Delta(w) & \stackrel{a d j_{s}}{\hookrightarrow} & \theta_{s} \Delta(w) & \rightarrow & \Delta(w s) & \text { if } w s \in W(\mathfrak{p}) \text { and } w s>w, \\
\Delta(w s) & \hookrightarrow & \theta_{s} \Delta(w) & \stackrel{a_{j} j^{s}}{\rightarrow} & \Delta(w) & \text { if } w s \in W(\mathfrak{p}) \text { and } w s<w, \\
\Delta(w) & \stackrel{a d j_{s}}{\hookrightarrow} & \theta_{s} \Delta(w) & \rightarrow & \Delta(w) & \text { if } w s \notin W(\mathfrak{p}) .
\end{array}
$$

In fact, the standard objects can also be realized as "thickened" Verma modules. In Theorem 3.1 we prove an equivalence of categories between $\mathcal{O}_{0}^{\mathfrak{p}-\text { pres }}$ and modules having a certain fixed singular central character inside the "thick" category $\mathcal{O}$ (i.e. an enlargement of $\mathcal{O}$ in the sense that the Cartan subalgebra acts locally nilpotent). It generalizes the equivalence from $[\mathrm{So3}$ to the singular situation. In this realization simple objects are well known. A disadvantage of this setup, however, is the fact that the usual definition and techniques of translation functors are not available. Thus we have two equivalent realizations of our category of Harish-Chandra bimodules: as a subcategory in $\mathcal{O}$ and as a subcategory of the thick category $\mathcal{O}$. Each realization has advantages and disadvantages. The most convenient description for us, however, is $\mathcal{O}_{0}^{\mathfrak{p}-\text { pres }}$ as both, the classical technique of translation functors and the restriction techniques (to subalgebras), are available in this context.

The principal series modules inside the categories of Harish-Chandra bimodules play an important role. Motivated by the results of Irving, which describe principal series modules as shuffled Verma modules (see [r1]), we define shuffled standard objects. We prove that shuffling defines an auto-equivalence of the bounded derived categories of $\mathcal{O}_{0}$ (Theorem [5.7) and $\mathcal{O}_{0}^{\mathfrak{p}-\text { pres }}$ (Theorem [5.9) respectively. This implies, in particular, that the shuffled standard objects are indecomposable. As one of our main results we show in Theorem 6.3 that the center of the universal enveloping algebra of $\mathfrak{g}$ maps naturally onto their endomorphism rings, which are all isomorphic to the coinvariant algebra associated with $W_{\mathfrak{p}}$. This is a generalization of [Be, Section 4] and [So2, Endomorphismensatz] since for $\mathfrak{p}=\mathfrak{g}$ the unique standard object in $\mathcal{O}_{0}^{\mathfrak{p}-\text { pres }}$ is the big projective module from $\mathcal{O}_{0}$. We also show that the socle of a standard module in $\mathcal{O}_{0}^{\mathfrak{p}-\text { pres }}$ is simple, a statement which is quite surprising from the point of view of Harish-Chandra bimodules.

Since a standard module, considered as an object in $\mathcal{O}_{0}$, has a Verma filtration, a shuffled standard module has a filtration with principal series modules. Its submodule structure is in general completely unknown. It is well known that shuffled 
Verma modules or principal series modules can be described as $\mathcal{L}(\Delta, \nabla)$ for some Verma module $\Delta$ and some dual Verma module $\nabla$. One would expect that replacing the (dual) Verma modules by (dual) standard modules one would get the shuffled standard objects. A character argument however shows that this is not true. The complexity of the submodule structure of shuffled standard objects mirrors the complexity of the submodule structure of tilting and projective objects, since we prove that the latter have filtrations, subquotients of which are isomorphic to certain shuffled standard objects (Theorem 5.15 and Theorem 5.16).

In $\left[\right.$ BGS] it was shown that the quasi-hereditary algebra associated with $\mathcal{O}_{0}$ is Koszul, in particular, graded. This defines a graded version of $\mathcal{O}_{0}$ and gives graded lifts of simple, projective and Verma modules. It even happens that translation functors admit a graded analogue, [St3]. In Sections 7 and 8 we work out the graded version of the category $\mathcal{O}_{0}^{\mathfrak{p}-p r e s}$, including graded lifts of standard objects, properly standard objects and simple objects. Proposition 8.7 shows that the properly stratified structure on $\mathcal{O}_{0}^{\mathfrak{p}-\text { pres }}$ is compatible with the grading.

Again, the behavior of the graded versions of standard modules under graded translations is similar to the behavior of the graded versions of Verma modules. A combinatorial description of this behavior is given by a parabolic Hecke module $\mathcal{M}_{\mathfrak{p}}$, defined in $[\mathrm{De}]$, in the following sense. The graded versions of the standard modules correspond to the standard basis of the parabolic Hecke module, and graded translation through the $s$-wall corresponds to the multiplication by the Kazhdan-Lusztig self-dual element $C_{s}$ of the Hecke algebra (Theorem 7.7). In this picture the graded projective modules correspond to the self-dual KazhdanLusztig-Deodhar basis of $\mathcal{M}_{\mathfrak{p}}$. In this way we obtain a categorification of $\mathcal{M}_{\mathfrak{p}}$ via the translation functors on $\mathcal{O}_{0}^{\mathfrak{p}-\text { pres }}$. The combinatorics, which we get here, are the same as the one obtained in the Koszul-dual situation (see So1 Remark 3.2 (2)]) of the categorification process described in $\mathrm{BFK}$ via projective functors on parabolic subcategories of $\mathcal{O}_{0}$ (see also [St2]).

We finish the paper with two detailed examples for the Lie algebra $\mathfrak{g}=\mathfrak{s l}(3, \mathbb{C})$.

\section{The CATEgory of $\mathfrak{p}$-PRESEntable Modules in $\mathcal{O}$}

2.1. General conventions. Throughout the paper we fix the ground field $\mathbb{C}$ of complex numbers. For a Lie algebra $\mathfrak{a}$ we denote by $U(\mathfrak{a})$ the universal enveloping algebra of $\mathfrak{a}$ and by $Z(\mathfrak{a})$ the center of $U(\mathfrak{a})$. If not otherwise stated, modules over any finite-dimensional associative algebra are always right modules, whereas modules over universal enveloping algebras are always left modules. For two modules, $M$ and $N$ say, the trace of $M$ in $N$, denoted by $\operatorname{Tr}_{M}(N)$ is the sum of all images of all homomorphisms from $M$ to $N$. For an abelian category $\mathcal{C}$ we denote by $[\mathcal{C}]$ the corresponding Grothendieck group, and for an exact functor $F$ on $\mathcal{C}$ we denote by $[F]$ the induced endomorphism of $[\mathcal{C}]$. For a module $M$ of some module category, $\mathcal{C}$ say, we denote by $[M]$ the image of $M$ in $[\mathcal{C}]$, and define the category $\operatorname{Add}(M)$ as the full subcategory of $\mathcal{C}$ consisting of all modules which admit a direct sum decomposition with summands being direct summands of $M$. We denote by ID the identity functor.

2.2. The category $\mathcal{O}$. Let $\mathfrak{g}$ denote a semi-simple finite-dimensional complex Lie algebra with a fixed triangular decomposition, $\mathfrak{g}=\mathfrak{n}_{-} \oplus \mathfrak{h} \oplus \mathfrak{n}_{+}$, where $\mathfrak{h}$ is a fixed Cartan subalgebra of $\mathfrak{g}$, and $\mathfrak{b}=\mathfrak{h} \oplus \mathfrak{n}_{+}$is the corresponding Borel subalgebra. 
This defines a set of simple roots $\pi \subset R$ inside the set of all roots. Let $W$ be the corresponding Weyl group with the length function $l$. By $e$ and $w_{0}$ we denote the shortest and the longest elements in $W$ respectively.

The Bernstein-Gelfand-Gelfand category $\mathcal{O}=\mathcal{O}(\mathfrak{g}, \mathfrak{b})$, associated with the above triangular decomposition of $\mathfrak{g}$, is defined as the full subcategory in the category of all $\mathfrak{g}$-modules which consists of all finitely generated, $\mathfrak{h}$-diagonalizable, and locally $U\left(\mathfrak{n}_{+}\right)$-finite $\mathfrak{g}$-modules (see $\left.\mathrm{BGG}\right)$. Important objects of $\mathcal{O}$ are Verma modules $M(\lambda)$, defined as follows: for $\lambda \in \mathfrak{h}^{*}$ we first define a $\mathfrak{b}$-module structure on $\mathbb{C}_{\lambda}=\mathbb{C}$ via $(h+n) v=\lambda(h) v$ for $h \in \mathfrak{h}, n \in \mathfrak{n}$, and $v \in \mathbb{C}$, and then set $M(\lambda)=U(\mathfrak{g}) \otimes_{U(\mathfrak{b})}$ $\mathbb{C}_{\lambda}$; see [Ja, Kapitel 4] (or [Di, Chapter 7]). The module $M(\lambda)$ has a unique simple quotient, which we will denote by $L(\lambda)$.

The action of the algebra $Z(\mathfrak{g})$ on every module from $\mathcal{O}$ is locally finite and thus, with respect to this action, the category $\mathcal{O}$ decomposes into a direct sum of full subcategories indexed by the maximal ideals in $Z(\mathfrak{g})$ :

$$
\mathcal{O}=\bigoplus_{\mathfrak{m} \in \operatorname{Max} Z(\mathfrak{g})} \mathcal{O}_{\mathfrak{m}}
$$

where

$$
\mathcal{O}_{\mathfrak{m}}=\left\{M \in \mathcal{O} \mid \exists k \in \mathbb{N}: \mathfrak{m}^{k} M=0\right\}
$$

is usually called a block of $\mathcal{O}$.

Any linear map $\lambda: \mathfrak{h} \rightarrow \mathbb{C}$ extends uniquely to an algebra homomorphism from the symmetric algebra $\mathcal{S}(\mathfrak{h})$ to $\mathbb{C}$. Via the Harish-Chandra homomorphism, this defines the maximal ideal $\mathfrak{m}_{\lambda}$ of $Z(\mathfrak{g})$. From now on we restrict our consideration to the trivial or principal block $\mathcal{O}_{0}=\mathcal{O}_{\mathfrak{m}_{0}}$. Because of Soergel's equivalence of categories ([So2 Theorem 11]) our restriction is equivalent to the condition that we consider a regular block of $\mathcal{O}$. The block $\mathcal{O}_{0}$ can alternatively be described as the block of $\mathcal{O}$, containing the trivial (one-dimensional) $\mathfrak{g}$-module $L(0)$. Simple modules in $\mathcal{O}_{0}$ are modules $\{L(w \cdot 0) \mid w \in W\}$, where $\cdot$ stands for the dot-action of $W$ on $\mathfrak{h}^{*}$. This action is defined as follows: $w \cdot \lambda=w(\lambda+\rho)-\rho$, where $\rho$ denotes the half-sum of all positive roots. To simplify our notation, we put $L(w)=L(w \cdot 0)$ and $M(w)=M(w \cdot 0)$. Every $M \in \mathcal{O}_{0}$ has finite length and for $w \in W$ we denote by $[M: L(w)]$ the multiplicity of $L(w)$ in $M$.

It is well known (see for example [BGG]) that $\mathcal{O}$ has enough projective modules. Let us denote by $P(w)$ the indecomposable projective cover and by $I(w)$ the indecomposable injective envelope of $L(w)$ respectively. By abstract nonsense, the category $\mathcal{O}_{0}$ is equivalent to the category of finitely generated right modules over the finite-dimensional associative algebra $A=\operatorname{End}_{\mathfrak{g}} P$, where $P=\bigoplus_{w \in W} P(w)$. The algebra $A$ is quasi-hereditary with respect to the Bruhat order $\leq$ on $W$, with Verma modules being the standard modules (see [CPS]). A filtration, whose subquotients are standard modules, is usually called a standard filtration. In particular, all projective modules in $\mathcal{O}$ have a standard filtration.

The Chevalley anti-involution $\omega$ gives rise to a duality on $\mathcal{O}$, that is a contravariant exact involutive equivalence preserving simple modules. This duality, which we will denote by (and write also $M^{*}$ instead of $(M)$ for $M \in \mathcal{O}$ ), is defined as follows: for $M \in \mathcal{O}$ the space $\operatorname{Hom}_{\mathbb{C}}(M, \mathbb{C})$ has a natural $\mathfrak{g}$-module structure given by $(g f)(m)=f(\omega(g) m)$ for $m \in M, g \in \mathfrak{g}$ and $f \in \operatorname{Hom}_{\mathbb{C}}(M, \mathbb{C})$. The maximal $\mathfrak{h}$-diagonalizable part $M^{\mathfrak{w}}=\operatorname{Hom}_{\mathbb{C}}(M, \mathbb{C})^{\mathfrak{h}-s s}$ of $\operatorname{Hom}_{\mathbb{C}}(M, \mathbb{C})$ is in fact a 
$\mathfrak{g}$-submodule and one checks that $M^{w} \in \mathcal{O}$. For all $w \in W$ one has $I(w) \cong P(w)^{\text {. }}$. The modules $M(w)^{*}, w \in W$, are called costandard modules in $\mathcal{O}_{0}$.

2.3. The category $\mathcal{O}_{0}^{\mathfrak{p}-\text { pres }}$ and its abelian structure. Now let $\mathfrak{p} \supseteq \mathfrak{b}$ be a parabolic subalgebra of $\mathfrak{g}$. Let $\mathfrak{p}=\mathfrak{a} \oplus \mathfrak{h}_{\mathfrak{a}}^{\perp} \oplus \mathfrak{n}$ be the Levi decomposition of $\mathfrak{p}$, where $\mathfrak{n}$ is the nilpotent radical, $\mathfrak{a} \oplus \mathfrak{h}_{\mathfrak{a}}^{\perp}$ is the reductive Levi factor, $\mathfrak{a}$ is semi-simple and $\mathfrak{h}_{\mathfrak{a}}^{\perp}$ is the center of $\mathfrak{a} \oplus \mathfrak{h}_{\mathfrak{a}}^{\perp}$. Denote by $W_{\mathfrak{p}}$ the Weyl group of $\mathfrak{a}$ with the longest element $w_{0}^{\mathfrak{p}}$. The set of all longest right coset representatives of $W$ modulo $W_{\mathfrak{p}}$ is denoted by $W(\mathfrak{p})$. Put $P_{\mathfrak{p}}=\bigoplus_{w \in W(\mathfrak{p})} P(w), I_{\mathfrak{p}}=\bigoplus_{w \in W(\mathfrak{p})} I(w)$, and $B=B_{\mathfrak{p}}=\operatorname{End}\left(P_{\mathfrak{p}}\right)$.

We define the category $\mathcal{O}_{0}^{\mathfrak{p}-\text { pres }}$ as the full subcategory of $\mathcal{O}_{0}$, which consists of all modules $M$, having a presentation $P_{1} \rightarrow P_{0} \rightarrow M \rightarrow 0$, with $P_{1}, P_{0} \in \operatorname{Add}\left(P_{\mathfrak{p}}\right)$. By $\left[\mathrm{Au}\right.$ Proposition 5.3], $\mathcal{O}_{0}^{\mathfrak{p}-\text { pres }}$ is equivalent to the category of finitely generated right $B$-modules; it is in fact equivalent to several other categories:

- Harish-Chandra bimodules with generalized trivial character from the lefthand side and certain singular character from the right-hand side (see [BG]).

- The full subcategory of $\mathcal{O}_{0}$, objects of which have a copresentation by modules in $\operatorname{Add}\left(I_{\mathfrak{p}}\right)$ (see Remark 2.4).

- The full subcategory of $\mathcal{O}_{0}$, objects of which are Enright-complete (see [En]) when restricted to $\mathfrak{a}$ (see KM1).

The last realization also connects $\mathcal{O}_{0}^{\mathfrak{p}-\text { pres }}$ to the parabolic generalizations $\mathcal{O}(\mathcal{P}, \Lambda)$ of $\mathcal{O}$, studied in FKM1. In particular, there is a connection to $\mathcal{S}$-subcategories of $\mathcal{O}$, studied in [FKM2], and to certain categories of Gelfand-Zetlin modules, considered in [KM2].

The subcategory $\mathcal{O}_{0}^{\mathfrak{p}-\text { pres }}$ inside $\mathcal{O}_{0}$ is obviously not closed by taking kernels and cokernels. However, via the equivalence to the category of $B$-modules, $\mathcal{O}_{0}^{\mathfrak{p}-\text { pres }}$ inherits an abelian structure. The inclusion $\mathfrak{i}: \mathcal{O}_{0}^{\mathfrak{p}-\text { pres }} \hookrightarrow \mathcal{O}_{0}$ is right exact but not left exact in general. The characterization of exact sequences in $\mathcal{O}_{0}^{\mathfrak{p}-\text { pres }}$ is quite natural and looks as follows:

Lemma 2.1. Let

$$
\ldots \stackrel{f_{i-2}}{\longrightarrow} M_{i-1} \stackrel{f_{i-1}}{\longrightarrow} M_{i} \stackrel{f_{i}}{\rightarrow} M_{i+1} \stackrel{f_{i+1}}{\longrightarrow} \ldots
$$

be a sequence of modules in $\mathcal{O}_{0}^{\mathfrak{p}-\text { pres }}$. Then the following conditions are equivalent:

(i) The sequence (2.1) is exact in $\mathcal{O}_{0}^{\mathfrak{p}-\text { pres }}$

(ii) We have $f_{i} \circ f_{i-1}=0$ and $\operatorname{Hom}_{\mathfrak{g}}\left(P_{\mathfrak{p}}, \operatorname{Ker}\left(f_{i}\right) / \operatorname{Im}\left(f_{i-1}\right)\right)=0$ for all $i$.

(iii) The induced sequence

$$
\ldots \stackrel{\tilde{f}_{i-2}}{\rightarrow} \operatorname{Hom}_{\mathfrak{g}}\left(P_{\mathfrak{p}}, M_{i-1}\right) \stackrel{\tilde{f}_{i-1}}{\rightarrow} \operatorname{Hom}_{\mathfrak{g}}\left(P_{\mathfrak{p}}, M_{i}\right) \stackrel{\tilde{f}_{i}}{\rightarrow} \operatorname{Hom}_{\mathfrak{g}}\left(P_{\mathfrak{p}}, M_{i+1}\right) \stackrel{\tilde{f}_{i+1}}{\rightarrow} \ldots
$$

is exact.

Proof. The equivalence (i) $\Leftrightarrow$ (iii) follows from the fact that $P_{\mathfrak{p}}$ is a projective generator of $\mathcal{O}_{0}^{\mathfrak{p}-\text { pres }}$. The equivalence with (ii) is then also straightforward.

Corollary 2.2. Let

$$
\ldots \stackrel{f_{i-2}}{\longrightarrow} M_{i-1} \stackrel{f_{i-1}}{\longrightarrow} M_{i} \stackrel{f_{i}}{\rightarrow} M_{i+1} \stackrel{f_{i+1}}{\longrightarrow} \ldots
$$

be an exact sequence of modules from $\mathcal{O}_{0}$, and assume that $M_{i} \in \mathcal{O}_{0}^{\mathfrak{p}-\text { pres }}$ for all $i$. Then the sequence (2.3) is also exact as the sequence of modules from $\mathcal{O}_{0}^{\mathfrak{p}-\text { pres }}$. 
Proof. The second condition of Lemma 2.1 is obviously satisfied.

Example 2.3. Let $\mathfrak{g}=\mathfrak{s l}(2, \mathbb{C})$ and $\mathfrak{p}=\mathfrak{g}$ with simple reflection $s$. We have a complex in $\mathcal{O}_{0}$ of the form

$$
0 \rightarrow M(e)^{*} \stackrel{f}{\rightarrow} P(s) \stackrel{g}{\rightarrow} M(e)^{*} \rightarrow 0,
$$

where $f, g \neq 0$, which is obviously not exact, but it is exact when considered as a sequence in $\mathcal{O}_{0}^{\mathfrak{p}-\text { pres }}$.

2.4. The category $\mathcal{O}_{0}^{\mathfrak{p}-\text { pres }}$ and coapproximation. According to $\mathrm{Au}$, Section 3] the inclusion functor $\mathfrak{i}$ has a right adjoint, say $\mathfrak{j}: \mathcal{O}_{0} \rightarrow \mathcal{O}_{0}^{\mathfrak{p}-\text { pres }}$, which we call the coapproximation with respect to $P_{\mathfrak{p}}$.

In general, coapproximation functors are defined as follows. Let $Q \in \mathcal{O}_{0}$ be projective and $M \in \mathcal{O}_{0}$. Let $p_{M}: M_{Q} \rightarrow \operatorname{Tr}_{Q}(M)$ be a projective cover. Then the coapproximation $\mathfrak{j}_{Q}$ with respect to $Q$ is defined on objects by $\mathfrak{j}_{Q}(M):=$ $M_{Q} / \operatorname{Tr}_{Q}\left(\operatorname{ker} p_{M}\right)$. Any $f \in \operatorname{Hom}_{\mathcal{O}_{o}}(M, N)$ can be restricted to a morphism $\operatorname{Tr}_{Q}(M)$ $\rightarrow \operatorname{Tr}_{Q}(N)$ and then lifted to a morphism $M_{Q} \rightarrow N_{Q}$, which maps $\operatorname{ker} p_{M}$ to $\operatorname{ker} p_{N}$. Hence factorization gives an element $\mathfrak{j}_{Q}(f) \in \operatorname{Hom}_{\mathfrak{g}}\left(\mathfrak{j}_{Q}(M), \mathfrak{j}_{Q}(N)\right)$. One has to check that all this is well defined and independent of the choices (see [Au, KM1]). Finally, this defines a functor $\mathfrak{j}_{Q}$ called coapproximation with respect to $Q$. In the following we only consider $\mathfrak{j}=\mathfrak{j}_{P_{\mathfrak{p}}}$.

Remark 2.4. Dually, one can define approximation with respect to a certain module and consider $\mathcal{O}_{0}^{\mathfrak{p}-\text { copres }}$, the full subcategory in $\mathcal{O}_{0}$ which consists of all modules $M$ having a copresentation $0 \rightarrow M \rightarrow I_{1} \rightarrow I_{2}$ with $I_{1}, I_{2} \in \operatorname{Add}\left(I_{\mathfrak{p}}\right)$. Duality induces an obvious contravariant equivalence $\mathcal{O}_{0}^{\mathfrak{p}-\text { copres }} \cong \mathcal{O}_{0}^{\mathfrak{p}-\text { pres }}$. Further, one can also show that approximation and coapproximation induce mutually inverse covariant equivalences between these two categories.

Associated to $\mathfrak{p}$ we have Zuckermann's functor $\mathfrak{z}=\mathfrak{z}_{\mathfrak{p}}$ which is defined as follows: for $M \in \mathcal{O}_{0}$ the module $\mathfrak{z}(M)$ is the maximal submodule of $M$ satisfying $\operatorname{Hom}_{\mathfrak{g}}\left(P_{\mathfrak{p}}, \mathfrak{z}(M)\right)=0$; the action on morphisms is given by restriction. Denote by $\mathfrak{z}=\mathfrak{z}$ the dual Zuckermann's functor. The next statement follows immediately from the definitions.

Lemma 2.5. There is the following exact sequence of functors on $\mathcal{O}_{0}$ :

$$
\mathfrak{i j} \stackrel{\mathfrak{x}}{\rightarrow} \mathrm{ID} \rightarrow \mathfrak{z}^{\text {*t }} \rightarrow 0 .
$$

Moreover, for every $M \in \mathcal{O}_{0}$ the kernel $K_{M}$ of the natural morphism $\mathfrak{i j}(M) \stackrel{\mathfrak{x}}{\rightarrow} M$ satisfies $\operatorname{Hom}_{\mathfrak{g}}\left(P_{\mathfrak{p}}, K_{M}\right)=0$.

Proposition 2.6. The functor $\star=\mathfrak{j}$ 站: $\mathcal{O}_{0}^{\mathfrak{p}-\text { pres }} \rightarrow \mathcal{O}_{0}^{\mathfrak{p}-\text { pres }}$ is a duality on $\mathcal{O}_{0}^{\mathfrak{p}-\text { pres }}$.

In the following we will also write $M^{\star}$ instead of $\star(M)$.

Proof. $\star$ is contravariant because is contravariant and $\mathfrak{i}, \mathfrak{j}$ are covariant. To show that $\star$ is exact, we consider a short exact sequence

$$
0 \stackrel{f_{0}}{\rightarrow} M_{1} \stackrel{f_{1}}{\rightarrow} M_{2} \stackrel{f_{2}}{\rightarrow} M_{3} \stackrel{f_{3}}{\rightarrow} 0
$$

in $\mathcal{O}_{0}^{\mathfrak{p}-\text { pres }}$. By Lemma 2.1 applying $\mathfrak{i}$ gives a complex in $\mathcal{O}$, whose homology has trivial $P_{\mathfrak{p}}$-trace. The latter is still true for the sequal sence, since is

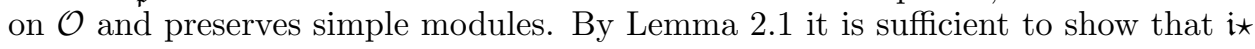


applied to (2.4) gives rise to a complex whose homology has trivial $P_{\mathfrak{p}}$-trace. Using Lemma 2.5 the sequence (2.4) gives rise to the following commutative diagram:

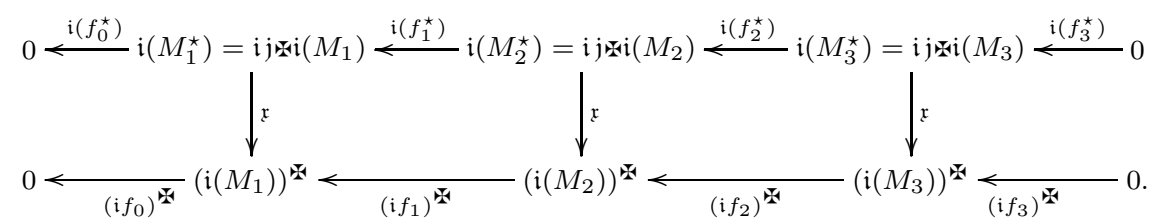

From Lemma 2.5 we get that the kernels and cokernels of the vertical maps have trivial $P_{\mathfrak{p}}$-trace. Hence the homology in the upper row has trivial $P_{\mathfrak{p}}$-trace and therefore $\star$ is exact.

The fact that $\star$ preserves simple objects in $\mathcal{O}_{0}^{\mathfrak{p}-\text { pres }}$ follows from Lemma 2.7 below. From Lemma 2.12 below it follows that $\star$ is involutive.

2.5. Projective, simple and standard objects in $\mathcal{O}_{0}^{\mathfrak{p}-\text { pres }}$. The projective modules $P(w) \in \mathcal{O}_{0}$, where $w \in W(\mathfrak{p})$, are actually objects in $\mathcal{O}_{0}^{\mathfrak{p}-\text { pres }}$. In fact, they constitute an exhaustive list of indecomposable projective objects in $\mathcal{O}_{0}^{\mathfrak{p}-\text { pres }}$. We define $S(w)=P(w) / \operatorname{Tr}_{P_{\mathfrak{p}}}(\operatorname{rad} P(w)) \in \mathcal{O}_{0}$. By construction it is even an object in $\mathcal{O}_{0}^{\mathfrak{p}-\text { pres }}$ where it is simple with projective cover isomorphic to $P(w)$.

Lemma 2.7. Let $w \in W(\mathfrak{p})$. Then the following assertions hold:

(1) The module $S(w) \in \mathcal{O}_{0}$ is indecomposable.

(2) The module $S(w) \in \mathcal{O}_{0}$ is simple if and only if $\mathfrak{p}=\mathfrak{b}$.

(3) As an object in $\mathcal{O}_{0}$ we have $[S(w): L(w)]=1$ and $\left[S(w): L\left(w^{\prime}\right)\right]=0$ for all $w^{\prime} \in W(\mathfrak{p}), w \neq w^{\prime}$.

(4) Considered as an object in $\mathcal{O}_{0}^{\mathfrak{p}-\text { pres }}$, there is an isomorphism $S(w)^{\star} \cong S(w)$ for all $w \in W(\mathfrak{p})$

Proof. The first and the third statements follow immediately from the definition of $S(w)$. For the second statement it is enough to show that $S(w)$ is not simple in the case $\mathfrak{p} \neq \mathfrak{b}$. But in this case one can find $w^{\prime} \in w W_{\mathfrak{p}}, w^{\prime} \neq w$, such that $\operatorname{rad} M\left(w^{\prime}\right)$ has a primitive element of weight $w \cdot 0$ and hence the module $L(w)$ on the top. This implies $\left[S(w): L\left(w^{\prime}\right)\right] \neq 0$ and hence $S(w)$ is not a simple $\mathfrak{g}$-module. To prove the last statement we consider $S(w)^{-} \in \mathcal{O}_{0}$. This module has a simple socle, isomorphic to $L(w)$, and $\operatorname{Hom}_{\mathfrak{g}}\left(P_{\mathfrak{p}}, S(w)^{\mathbb{w}} / L(w)\right)=0$ by the third statement. From the definition of $\mathfrak{j}$ we now get $\mathfrak{j}\left(S(w)^{w}\right) \cong P(w) / \operatorname{Tr}_{P_{\mathfrak{p}}}(\operatorname{rad} P(w)) \cong S(w)$, completing the proof.

For $w \in W(\mathfrak{p})$ we consider the module $P^{w}=\bigoplus_{w^{\prime}} P\left(w^{\prime}\right)$, where $w^{\prime} \in W(\mathfrak{p})$ and $w^{\prime}<w$, and set $\Delta(w)=P(w) / \operatorname{Tr}_{P w}(P(w)) \in \mathcal{O}_{0}$. It is in fact an object in $\mathcal{O}_{0}^{\mathfrak{p}-\text { pres }}$ (which will be denoted by the same symbol). In particular, $\Delta\left(w_{0}^{\mathfrak{p}}\right)=$ $P\left(w_{0}^{\mathfrak{p}}\right)$ in $\mathcal{O}_{0}^{\mathfrak{p}-\text { pres }}$. The modules $\Delta(w)$ are called standard modules because of their role in the properly stratified structure on $\mathcal{O}_{0}^{\mathfrak{p}-\text { pres }}$, which will be explained later. These modules have a universal property, analogous to that of Verma modules. To formulate this we need one more notion. We call $M \in \mathcal{O}_{0}^{\mathfrak{p}-\text { pres }}$ a $\mathfrak{p}$-highest weight module with $\mathfrak{p}$-highest weight $w \in W(\mathfrak{p})$ provided that $M$ is generated by some $v \in M$ of weight $w \cdot 0$ such that $\mathfrak{n} v=0$. For a $\mathfrak{p}$-highest weight module we have:

Lemma 2.8. Every $\mathfrak{p}$-highest weight module with the $\mathfrak{p}$-highest weight $w \in W(\mathfrak{p})$ is a homomorphic image of $\Delta(w)$. 
Proof. Let $M \in \mathcal{O}_{0}^{\mathfrak{p}-\text { pres }}$ be a p-highest weight module with $\mathfrak{p}$-highest weight $w \in$ $W(\mathfrak{p})$. Since $M$ is generated by $v$ and $\mathfrak{n} v=0$, there is an epimorphism, $\varphi$ say, from $P(w)$ to $M$, sending the canonical generator of $P(w)$ to $v$. But $\mathfrak{n} v=0$ even implies that $\operatorname{Tr}_{P} w(P(w))$ is annihilated by $\varphi$. Hence, $M$ is a homomorphic image of $\Delta(w)$.

We denote by $P^{\mathfrak{a}}(w \cdot 0)$ the projective cover in the category $\mathcal{O}(\mathfrak{a}, \mathfrak{a} \cap \mathfrak{b})$ of the simple highest weight module with highest weight $w \cdot 0$. The $\mathfrak{p}$-module structure on $P^{\mathfrak{a}}(w \cdot 0)$ is defined via $\mathfrak{n} P^{\mathfrak{a}}(w \cdot 0)=0$.

Proposition 2.9. Let $w \in W(\mathfrak{p})$.

(1) As $\mathfrak{g}$-modules, $\Delta(w) \cong U(\mathfrak{g}) \otimes_{U(\mathfrak{p})} P^{\mathfrak{a}}(w \cdot 0)$.

(2) As an $\mathfrak{a}$-module, the module $\Delta(w)$ is a direct sum of self-dual projective modules from $\mathcal{O}(\mathfrak{a}, \mathfrak{a} \cap \mathfrak{b})$.

(3) As an $\mathfrak{a}$-module, the module $P(w)$ is a direct sum of self-dual projective modules from $\mathcal{O}(\mathfrak{a}, \mathfrak{a} \cap \mathfrak{b})$.

Proof. Since the module $N=U(\mathfrak{g}) \otimes_{U(\mathfrak{p})} P^{\mathfrak{a}}(w \cdot 0)$ is generated by an element of weight $w \cdot 0$, which is annihilated by $\mathfrak{n}$, we can apply Lemma2.8 and get a surjection from $\Delta(w)$ onto $N$. On the other hand, using the adjunction

$$
\operatorname{Hom}_{\mathfrak{g}}\left(U(\mathfrak{g}) \otimes_{U(\mathfrak{p})} P^{\mathfrak{a}}(w \cdot 0),{ }_{-}\right) \cong \operatorname{Hom}_{\mathfrak{p}}\left(P^{\mathfrak{a}}(w \cdot 0), \operatorname{Res}_{U(\mathfrak{p})}(-)\right)
$$

and the projectivity of $P^{\mathfrak{a}}(w \cdot 0)$, one gets a universal property of $N$, analogous to that of $\Delta(w)$ given by Lemma 2.8. Therefore, $\Delta(w)$ is a homomorphic image of $N$ and thus $N \cong \Delta(w)$ and the first statement is proved.

Since $w \in W(\mathfrak{p})$, the module $P^{\mathfrak{a}}(w \cdot 0)$ is the projective cover of a simple Verma module, and therefore self-dual. Further, $U(\mathfrak{g})$ is a direct sum of finite-dimensional $\mathfrak{a}$-modules under the adjoint action. Hence, as an $\mathfrak{a}$-module, the module $U(\mathfrak{g}) \otimes_{U(\mathfrak{p})}$ $P^{\mathfrak{a}}(w \cdot 0)$ is isomorphic to a direct sum of modules of the form $E \otimes P^{\mathfrak{a}}(w \cdot 0)$, where $E$ is a finite-dimensional $\mathfrak{a}$-module. Since a self-dual projective object stays both projective and self-dual after tensoring with a finite-dimensional module, the second statement follows.

The last statement is now evident, because $P(w)$ is a direct summand of some $E \otimes P\left(w_{0}^{\mathfrak{p}}\right) \cong E \otimes \Delta\left(w_{0}^{\mathfrak{p}}\right)$, where $E$ is a finite-dimensional $\mathfrak{a}$-module.

Proposition 2.10. Let $M \in \mathcal{O}_{0}$ be a module which, viewed as an $\mathfrak{a}$-module, is a direct sum of self-dual projective modules in $\mathcal{O}(\mathfrak{a}, \mathfrak{a} \cap \mathfrak{b})$. Then $M \in \mathcal{O}_{0}^{\mathfrak{p}-\text { pres }}$.

Proof. Recall that self-dual projectives are exactly the projective covers of simple Verma modules (【זr2]). Hence, as an $\mathfrak{a}$-module (and then of course also as a $\mathfrak{g}$ module), $M$ is generated by elements of weight $x \cdot 0$, where $s x<x$ for any simple reflection $s \in W_{\mathfrak{p}}$ (i.e. $x \cdot 0$ is an $\mathfrak{a}$-anti-dominant weight). The projective cover $P_{M}$ of $M$ in $\mathcal{O}_{0}$ is a direct sum of modules $P(w), w \in W(\mathfrak{p})$, and hence $P_{M} \in \operatorname{Add}\left(P_{\mathfrak{p}}\right)$. As both, $M$ and $P_{M}$, are direct sums of self-dual projective $\mathfrak{a}$-modules (see the last statement of Proposition [2.9), the kernel $K \hookrightarrow P_{M} \rightarrow M$ is also a direct sum of self-dual projective a-modules. As already proved, the projective cover of $K$ must belong to $\operatorname{Add}\left(P_{\mathfrak{p}}\right)$ and thus the module $M$ is presented by modules from $\operatorname{Add}\left(P_{\mathfrak{p}}\right)$. This completes the proof. 
In particular, Propositions 2.9 and 2.10 imply:

Corollary 2.11. The modules $\nabla(w)=\Delta(w)^{\text {and }} I(w) \cong P(w)^{\text {belong to }}$ $\mathcal{O}_{0}^{\mathfrak{p}-\text { pres }}$ for all $w \in W(\mathfrak{p})$.

From Corollary 2.11 we derive that the indecomposable injective objects in $\mathcal{O}_{0}^{\mathfrak{p}-\text { pres }}$ are precisely the injective modules $I(w), w \in W(\mathfrak{p})$. The modules $\nabla(w)$ are called costandard modules, because of their role in the properly stratified structure which will be explained in Subsection 2.6. First, however, we would like to finish the proof of Proposition 2.6.

Lemma 2.12. The functor $\star \star$ is isomorphic to ID on $\mathcal{O}_{0}^{\mathfrak{p}-\text { pres }}$.

Proof. We have $P_{\mathfrak{p}}^{\star} \in \mathcal{O}_{0}^{\mathfrak{p}-\text { pres }}$ by Corollary 2.11 and thus $P_{\mathfrak{p}}^{\star}=\mathfrak{j}\left(P_{\mathfrak{p}}^{\star}\right)=P_{\mathfrak{p}}^{\star}$. Therefore, $\star$ is isomorphic to on $\operatorname{Add}\left(P_{\mathfrak{p}}\right)$ and thus $\star \star$ is isomorphic to ID on $\operatorname{Add}\left(P_{\mathfrak{p}}\right)$. The latter functors must be isomorphic on the entire category $\mathcal{O}_{0}^{\mathfrak{p}-\text { pres }}$, since both functors are exact and $P_{\mathfrak{p}}$ is a projective generator of $\mathcal{O}_{0}^{\mathfrak{p}-\text { pres }}$.

Recall that a Verma flag of a module in $\mathcal{O}_{0}$ is a filtration, whose subquotients are isomorphic to Verma modules. As an interesting implication from the above results we get

Proposition 2.13. Let $M \in \mathcal{O}_{0}$. Then the following conditions are equivalent:

(i) $M$ has a filtration, subquotients of which are isomorphic to modules $\Delta(w)$, $w \in W(\mathfrak{p})$.

(ii) $M$ has a Verma flag and, viewed as an $\mathfrak{a}$-module, it is a direct sum of self-dual projective modules in $\mathcal{O}(\mathfrak{a}, \mathfrak{b} \cap \mathfrak{a})$.

(iii) $M$ is $\mathfrak{n}_{-}$-free and, viewed as an $\mathfrak{a}$-module, it is a direct sum of self-dual projective modules in $\mathcal{O}(\mathfrak{a}, \mathfrak{b} \cap \mathfrak{a})$.

(iv) For all $w \in W$ we have $\operatorname{Ext}_{\mathcal{O}}^{1}\left(M, M(w)^{-}\right)=0$, and, viewed as an $\mathfrak{a}$-module, $M$ is a direct sum of self-dual projective modules in $\mathcal{O}(\mathfrak{a}, \mathfrak{b} \cap \mathfrak{a})$.

Proof. The equivalence (iii) $\Leftrightarrow$ (iv) is well known; see for example [Rin, Section 4]. The equivalence (iii) $\Leftrightarrow$ (iii) follows from the standard fact that a module in $\mathcal{O}$ has a Verma flag if and only if it is $\mathfrak{n}_{-}$-free; see for example [Di, Chapter 7]. The implication (ii) $\Rightarrow$ (iii) is trivial and the inverse implication follows from Proposition 2.9 by induction on the length of a Verma filtration.

We will say that the module $M \in \mathcal{O}_{0}^{\mathfrak{p}-\text { pres }}$ (or $M \in \mathcal{O}_{0}$ ) has a standard filtration or standard flag provided that there is a filtration of $M$, whose subquotients are isomorphic to standard modules. Analogously, one defines costandard filtrations or costandard flags. We denote by $\mathcal{F}(\Delta)$ (resp. $\mathcal{F}(\nabla))$ the full subcategory in $\mathcal{O}_{0}^{\text {p-pres }}$ consisting of all modules, having a standard (resp. costandard) filtration. Note that if a module in $\mathcal{O}_{0}^{\mathfrak{p}-\text { pres }}$, considered as an object in $\mathcal{O}_{0}$, has a standard filtration, then (by Corollary 2.2) it also has a standard filtration, considered as an object in $\mathcal{O}_{0}^{\mathfrak{p}-\text { pres }}$.

Corollary 2.14. The full subcategory $\mathcal{F}(\Delta)$ of $\mathcal{O}_{0}$ is closed under taking direct summands.

Proof. Since the last condition in Proposition 2.13 is invariant under taking direct summands, the statement follows. 
2.6. Properly stratified structure on $\mathcal{O}_{0}^{\mathfrak{p}-\text { pres }}$. First, we revise the properly stratified structure on $\mathcal{O}_{0}^{\mathfrak{p}-\text { pres }}$ established in [FKM1]. We recall (see [D1]) that a finite-dimensional associative algebra $A$ with a fixed order $\leq$ on the set $\Lambda$ of isomorphism classes of simple $A$-modules (the latter will be denoted by $S(i), i \in \Lambda$ ) is called properly stratified provided that there exist some $A$-modules $\Delta(i)$ and $\bar{\Delta}(i)$, $i \in \Lambda$, such that

(PS1) The projective cover $P(i)$ of $S(i)$ surjects onto $\Delta(i)$ and the kernel of this surjection has a filtration, subquotients of which are isomorphic to $\Delta(j)$ with $j<i$.

(PS2) The module $\bar{\Delta}(i)$ surjects onto $S(i)$ and the kernel of this surjection has a filtration, subquotients of which are isomorphic to $S(j)$ with $j>i$.

(PS3) The module $\Delta(i)$ has a filtration subquotients of which are isomorphic to $\bar{\Delta}(i)$.

The modules $\Delta(i)$ are called standard modules and the modules $\bar{\Delta}(i)$ are called proper standard modules.

Remark 2.15. In the definition above we have chosen the order, opposite to the classical order on properly stratified algebras (as for example in [D1]).

For $w \in W(\mathfrak{p})$ we define the a-module $S^{\mathfrak{a}}(w)=P^{\mathfrak{a}}(w \cdot 0) / \operatorname{Tr}_{P^{\mathfrak{a}}(w \cdot 0)}\left(\operatorname{rad} P^{\mathfrak{a}}(w \cdot 0)\right)$ and then $\bar{\Delta}(w)=U(\mathfrak{g}) \otimes_{U(\mathfrak{p})} S^{\mathfrak{a}}(w)$, where the $\mathfrak{p}$-module structure on $S^{\mathfrak{a}}(w)$ is defined via $\mathfrak{n} S^{\mathfrak{a}}(w)=0$. For a simple, projective or standard object $M \in \mathcal{O}_{0}^{\mathfrak{p}-\text { pres }}$ we denote the $B=\operatorname{End}\left(P_{\mathfrak{p}}\right)$-module $\operatorname{Hom}_{\mathfrak{g}}\left(\operatorname{End}\left(P_{\mathfrak{p}}\right), M\right)$ with the same symbol by abuse of notation.

We consider the set $\{S(w): w \in W(\mathfrak{p})\}$ of iso-classes of simple $B$-modules with the order induced from the Bruhat order on $W$. The following theorem shows that there is no confusion with the notation $\Delta(w)$ we use for standard modules in $\mathcal{O}_{0}^{\mathfrak{p}-\text { pres }}$.

Theorem 2.16. The algebra $B$ is properly stratified with modules $\Delta(w)$ and $\bar{\Delta}(w)$, $w \in W(\mathfrak{p})$, being the standard and proper standard modules respectively.

Proof. (PS1) follows from Propositions [2.13] and [2.9. (PS2) follows from the construction of $\bar{\Delta}(w)$ and the universal property of the tensor product. Finally, (PS3) follows from (PS2) and exactness of the tensor induction from $\mathfrak{p}$ to $\mathfrak{g}$, considering any filtration $0=N_{0} \subset M_{1} \subset \cdots \subset N_{l}=P^{\mathfrak{a}}(w \cdot 0)$ such that $P^{\mathfrak{a}}(w \cdot 0) \rightarrow M_{i} / M_{i-1}$ and $\operatorname{dim} \operatorname{Hom}_{\mathfrak{a}}\left(P^{\mathfrak{a}}(w \cdot 0), M_{i} / M_{i-1}\right)=1$ for all $i$.

Remark 2.17. The proper standard modules $\bar{\Delta}(w)$ can be constructed in many different ways. We have defined them via parabolic induction. On the other hand, $\bar{\Delta}(w) \cong \Delta(w) / \operatorname{Tr}_{\Delta(w)}(\operatorname{rad} \Delta(w))$ and is also isomorphic to the module $\Delta(w) / R \Delta(w)$, where $R=\operatorname{rad}\left(\operatorname{End}_{\mathfrak{g}}(\Delta(w))\right)$. As an object in $\mathcal{O}_{0}$, the module $\bar{\Delta}(w)$ is isomorphic to the shuffled Verma module $M\left(w, w_{0}^{\mathfrak{p}}\right)$ in the sense of [Ir1], or to the corresponding twisted Verma module in the sense of $\mathrm{AL}$.

We define the proper costandard modules as $\bar{\nabla}(w) \cong \bar{\Delta}(w)^{\star}$ for all $w \in W(\mathfrak{p})$. One can also define properly stratified algebras in a dual way using injective modules and introducing costandard and proper costandard modules. The corresponding axioms will be dual with respect to those, described in (PS1), (PS2) and (PS3). We also remark that, although $\Delta(w)^{\star} \cong \Delta(w)^{\star}$, there is no $\mathfrak{g}$-module isomorphism $\bar{\Delta}(w)^{\star} \cong \bar{\Delta}(w)^{*}$ in general. 
Considering $\Delta(w)$ as an object in $\mathcal{O}_{0}$, we have the following statement:

Proposition 2.18. Let $w \in W(\mathfrak{p})$. The module $\Delta(w) \in \mathcal{O}_{0}$ has a Verma flag of length $\left|W_{\mathfrak{p}}\right|$. The subquotients in this filtration are exactly the modules $M\left(w^{\prime}\right)$, $w^{\prime} \in W_{\mathfrak{p}} w$, each occurring once.

Remark 2.19. Applying the non-exact functor $\mathfrak{j}$ to any Verma flag, given by Proposition 2.18 one gets a filtration of the standard module $\Delta(w)$ in which all subquotients are isomorphic to the proper standard module $\bar{\Delta}(w)$. This is exactly the filtration, required by (PS3).

Proof. We write $\Delta(w) \cong U(\mathfrak{g}) \otimes_{U(\mathfrak{p})} P^{\mathfrak{a}}(w \cdot 0)$ with the notation of Proposition 2.9 The BGG-reciprocity implies that the self-dual projective module $P^{\mathfrak{a}}(w \cdot 0) \in$ $\mathcal{O}(\mathfrak{a}, \mathfrak{a} \cap \mathfrak{b})$ has a Verma flag of length $\left|W_{\mathfrak{p}}\right|$ with subquotients $M^{\mathfrak{a}}\left(w^{\prime}\right), w^{\prime} \in W_{\mathfrak{p}} w$, each occurring exactly once. Applying the exact functor $U(\mathfrak{g}) \otimes_{U(\mathfrak{p})}$ - to this filtration produces the desired filtration.

\section{An ALternative DeSCRIPTION OF $\mathcal{O}_{0}^{\mathfrak{p}-\text { pres }}$ VIA THE THICK CATEGORY $\mathcal{O}$}

The ideas and results of So3 can be used to give an alternative description of the category $\mathcal{O}_{0}^{\mathfrak{p}-\text { pres }}$ in terms of certain categories of Harish-Chandra bimodules and the "thickened" version of the category $\mathcal{O}$. The advantage of this approach is that in the latter categories the notion of a simple object coincides with the notion of a simple $\mathfrak{g}$-bimodule and a simple $\mathfrak{g}$-module respectively. The promised alternative description requires some new definitions and notions.

3.1. Thick category $\mathcal{O}$. For a $\mathfrak{g}$-module $M$, an integer $i \in\{1,2,3, \ldots\}$, and $\mu \in \mathfrak{h}^{*}$ we denote by

$$
M_{\mu}^{i}=\left\{v \in M \mid(h-\mu(h))^{i} v=0 \text { for all } h \in \mathfrak{h}\right\}
$$

the corresponding generalized weight space of exponent $i$. Note that $M_{\mu}^{i} \subset M_{\mu}^{i+1}$ by definition. Set $M_{\mu}^{\infty}=\bigcup_{i \geq 1} M_{\mu}^{i}$.

Let $\lambda \in \mathfrak{h}^{*}$ be dominant. For $i \in\{1,2,3, \ldots\} \cup\{\infty\}$ we define the $i$-thickened category $\mathcal{O}_{\lambda}^{i}$ as the full subcategory in the category of all $\mathfrak{g}$-modules, which consists of all $\mathfrak{g}$-modules $M$, which

- are locally b-finite;

- are annihilated by $\mathfrak{m}_{\lambda}^{m}, m \gg 0$;

- admit a decomposition $M=\bigoplus_{\mu \in \mathfrak{h}^{*}} M_{\mu}^{i}$.

We remark that $\mathcal{O}_{\lambda}^{1}=\mathcal{O}_{\lambda}$ is just the corresponding block of the classical category $\mathcal{O}$. One also has $\mathcal{O}_{\lambda}^{\infty}=\bigcup_{i \geq 1} \mathcal{O}_{\lambda}^{i}$. The category $\bigoplus_{\lambda} \mathcal{O}_{\lambda}^{\infty}$ is called the thick category $\mathcal{O}$. However, the main object of our interest will be the subcategory $\overline{\mathcal{O}_{\lambda}}$ of $\mathcal{O}_{\lambda}^{\infty}$, which is defined as the full subcategory of $\mathcal{O}_{\lambda}^{\infty}$, consisting of all modules $M$ annihilated by $\mathfrak{m}_{\lambda}$. (For $\lambda$ regular, this category was denoted by $\mathcal{O}_{\lambda}^{\prime}$ in [So3]). In Subsection 3.3 we will show that there exists $\lambda \in \mathfrak{h}^{*}$ such that the categories $\mathcal{O}_{0}^{\mathfrak{p}-\text { pres }}$ and $\overline{\mathcal{O}_{\lambda}}$ are equivalent.

3.2. Harish-Chandra bimodules. Let $\mathbf{H}$ denote the category of all finitelygenerated $U(\mathfrak{g})$-bimodules, which decompose into a direct sum of finite-dimensional $\mathfrak{g}$-modules under the adjoint action of $\mathfrak{g}$. Such bimodules are called Harish-Chandra bimodules. For dominant $\lambda, \mu$ and $i, j \in\{1,2,3, \ldots\} \cup\{\infty\}$ we define ${ }_{\lambda}^{i} \mathbf{H}_{\mu}^{j}$ as the full subcategory of $\mathbf{H}$ which consists of all bimodules $M$ such that $\mathfrak{m}_{\lambda}^{i} M=0$ if 
$i<\infty\left(\mathfrak{m}_{\lambda}^{k} M=0\right.$ for $k \gg 0$ if $\left.i=\infty\right)$ and $M \mathfrak{m}_{\mu}^{j}=0$ if $j<\infty\left(M \mathfrak{m}_{\mu}^{k}=0\right.$ for $k \gg 0$ otherwise). For $\mathfrak{g}$-modules $M$ and $N$, the space $\operatorname{Hom}_{\mathbb{C}}(M, N)$ becomes a $\mathcal{U}(\mathfrak{g})$-bimodule in a natural way $([\mathrm{Ja}, 6.2])$. Let $\mathcal{L}(M, N)$ denote its largest submodule contained in $\mathbf{H}$ (see Di, Proposition 1.7.9]). We refer the reader to [Ja] for the standard properties of these categories.

\subsection{An alternative description of $\mathcal{O}_{0}^{\mathfrak{p}-\text { pres }}$.}

Theorem 3.1. Let $\lambda$ be dominant and integral such that the stabilizer $W_{\lambda}$ of $\lambda$ in $W$ coincides with $W_{\mathfrak{p}}$. Then there is an equivalence of categories $\mathcal{O}_{0}^{\mathfrak{p}-\text { pres }} \stackrel{\sim}{\longrightarrow} \overline{\mathcal{O}_{\lambda}}$.

Proof. [BG, Theorem 5.9] provides an equivalence, $\beta:{ }_{0}^{\infty} \mathbf{H}_{0}^{1} \stackrel{\sim}{\longrightarrow} \mathcal{O}_{0}$, which is extended in [So3] to an equivalence $\bar{\beta}:{ }_{0}^{\infty} \mathbf{H}_{0}^{\infty} \stackrel{\sim}{\longrightarrow} \mathcal{O}_{0}$. Denote by $\eta:{ }_{0}^{\infty} \mathbf{H}_{0}^{1} \stackrel{\sim}{\longrightarrow}{ }_{0}^{1} \mathbf{H}_{0}^{\infty}$ the equivalence, obtained by interchanging the left and the right actions. It is shown in [So3] that, restricting $\bar{\beta}$ to ${ }_{0}^{1} \mathbf{H}_{0}^{\infty}$, gives an equivalence, $\gamma$, which induces an equivalence, $\varepsilon$ say, such that the following diagram commutes:

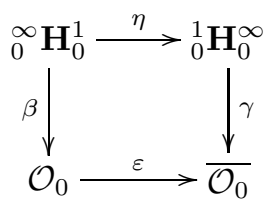

On the other hand, [BG, Theorem 5.9] provides an equivalence $\alpha:{ }_{0}^{\infty} \mathbf{H}_{\lambda}^{1} \stackrel{\sim}{\longrightarrow}$ $\mathcal{O}_{0}^{\mathfrak{p}-\text { pres }}$. The inclusion $\mathfrak{i}: \mathcal{O}_{0}^{\mathfrak{p}-\text { pres }} \hookrightarrow \mathcal{O}_{0}$ defines an inclusion, $\mathfrak{i}^{\prime}$ say, such that the following diagram commutes:

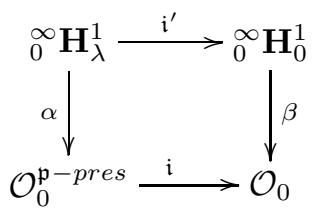

Denote by $\theta^{l}:{ }_{0}^{1} \mathbf{H}_{0}^{\infty} \rightarrow{ }_{\lambda}^{1} \mathbf{H}_{0}^{\infty}$ and $\theta^{r}:{ }_{0}^{\infty} \mathbf{H}_{0}^{1} \rightarrow{ }_{0}^{\infty} \mathbf{H}_{\lambda}^{1}$ the left and the right translations from 0 to $\lambda$ respectively. Since $\theta^{l}$ commutes naturally with tensoring over $\mathcal{U}(\mathfrak{g})$ with thick Verma modules from the right-hand side, the last commutative diagram extends to the following:

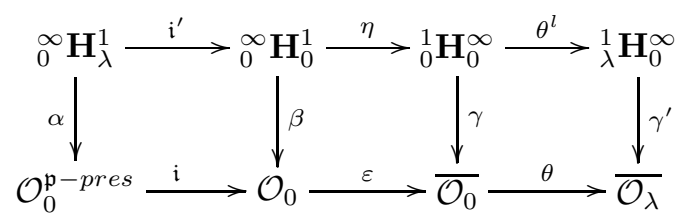

where $\theta: \overline{\mathcal{O}_{0}} \rightarrow \overline{\mathcal{O}_{\lambda}}$ is the translation from 0 to $\lambda$ and $\gamma^{\prime}$ is the induced equivalence, making the diagram commutative.

Lemma 3.2. $\theta^{l} \eta \mathfrak{i}^{\prime} \cong \eta$. 
Proof. Let $X \in{ }_{0}^{\infty} \mathbf{H}_{\lambda}^{1}$. We have

$$
\begin{aligned}
\theta^{l} \eta \mathfrak{i}^{\prime}(X) & \cong \theta^{l} \eta\left(\mathcal{L}\left(M(0), X \otimes_{U(\mathfrak{g})} M(\lambda)\right)\right) & & (\text { by [3.2) and [BG] Theorem 5.9]) } \\
& \cong \eta \theta^{r}\left(\mathcal{L}\left(M(0), X \otimes_{U(\mathfrak{g})} M(\lambda)\right)\right) & & \left(\text { as } \theta^{l}=\eta \theta^{r} \eta\right) \\
& \cong \eta\left(\mathcal{L}\left(M(\lambda), X \otimes_{U(\mathfrak{g})} M(\lambda)\right)\right) & & (\text { by [Ja] 6.33]) } \\
& \cong \eta(X) & & (\text { by [BG, Theorem 5.9]) }
\end{aligned}
$$

The isomorphisms above are all natural and the statement follows.

Define $F=\theta \varepsilon \mathfrak{i}: \mathcal{O}_{0}^{\mathfrak{p}-\text { pres }} \rightarrow \overline{\mathcal{O}_{\lambda}}$ and $G=\alpha \eta^{-1}\left(\gamma^{\prime}\right)^{-1}: \overline{\mathcal{O}_{\lambda}} \rightarrow \mathcal{O}_{0}^{\mathfrak{p}-\text { pres }}$. Then we have

$$
\begin{aligned}
& G F \cong \alpha \eta^{-1}\left(\gamma^{\prime}\right)^{-1} \theta \varepsilon \mathfrak{i} \\
& \cong \alpha \eta^{-1}\left(\gamma^{\prime}\right)^{-1} \theta \varepsilon \beta \mathfrak{i}^{\prime} \alpha^{-1} \quad \text { (by (3.2)) } \\
& \cong \alpha \eta^{-1}\left(\gamma^{\prime}\right)^{-1} \theta \gamma \eta \mathfrak{i}^{\prime} \alpha^{-1} \quad \text { (by (3.1)) } \\
& \cong \alpha \eta^{-1}\left(\gamma^{\prime}\right)^{-1} \gamma^{\prime} \theta^{l} \eta \mathfrak{i}^{\prime} \alpha^{-1} \text { (by (3.3)) } \\
& \cong \alpha \eta^{-1} \mathrm{ID} \eta \alpha^{-1} \quad \text { (by Lemma 3.2) } \\
& \cong \mathrm{ID} \text {, }
\end{aligned}
$$

and

$$
\begin{array}{rlrl}
F G & \cong \theta \varepsilon \mathfrak{i} \alpha \eta^{-1}\left(\gamma^{\prime}\right)^{-1} & \\
& \cong \theta \gamma \eta \mathfrak{i}^{\prime} \eta^{-1}\left(\gamma^{\prime}\right)^{-1} & & (\text { by }(3.3)) \\
& \cong \gamma^{\prime} \theta^{l} \eta \mathfrak{i}^{\prime} \eta^{-1}\left(\gamma^{\prime}\right)^{-1} & & \text { (by (3.3) }) \\
& \cong \gamma^{\prime} \eta \eta^{-1}\left(\gamma^{\prime}\right)^{-1} & & (\text { by Lemma 3.2) } \\
& \cong \text { ID. } & &
\end{array}
$$

Hence $F$ defines an equivalence of categories with inverse $G$.

Remark 3.3. The equivalence, given by Theorem 3.1 sends the proper standard module $\bar{\Delta}(w)$ in $\mathcal{O}_{0}^{\mathfrak{p}-\text { pres }}$ to the usual Verma module $M\left(w^{-1} \cdot \lambda\right)$ in $\overline{\mathcal{O}_{\lambda}}$ and the standard module $\Delta(w)$ in $\mathcal{O}_{0}^{\mathfrak{p}-\text { pres }}$ to the "maximally thickened" extension of the corresponding Verma module in $\overline{\mathcal{O}_{\lambda}}$. The existence of the duality on $\mathcal{O}_{0}^{\mathfrak{p}-\text { pres }}$ also follows now easily from the usual duality on $\overline{\mathcal{O}_{\lambda}}$.

\section{Standard MOdUles in $\mathcal{O}_{0}^{\mathfrak{p}-\text { pres }}$ AND Their translations}

In this section we study the behavior of standard modules in the category $\mathcal{O}_{0}^{\mathfrak{p}-\text { pres }}$ under translation functors. For a simple reflection $s$ we denote by $\theta_{s}: \mathcal{O} \rightarrow \mathcal{O}$ the translation functor through the $s$-wall. The functor $\theta_{s}$ is self-adjoint, and we have natural transformations $\operatorname{adj}_{s}:$ ID $\rightarrow \theta_{s}$ and $\left(\mathrm{adj}^{s}\right): \theta_{s} \rightarrow$ ID. To simplify notation, the adjunction morphisms $M \stackrel{a d j_{s}(M)}{\longrightarrow} \theta_{s} M$ and $\theta_{s} M \stackrel{a d j^{s}(M)}{\longrightarrow} M$ will be denoted by $M \stackrel{a^{a d j} s}{\longrightarrow} \theta_{s} M$ and $\theta_{s} M \stackrel{a d j^{s}}{\longrightarrow} M$ respectively. From Proposition 2.18 one gets that the adjunction morphism $\Delta(w) \stackrel{a d j_{s}}{\longrightarrow} \theta_{s} \Delta(w)$ is injective. We refer the reader to Ja] and [GJ Section 3] for details.

In what follows we will often use the following statement.

Lemma 4.1. Let $M \in \mathcal{O}_{0}$ be $\mathfrak{n}_{-}$-free and, as $\mathfrak{a}$-module, a direct sum of self-dual projective modules in $\mathcal{O}(\mathfrak{a}, \mathfrak{a} \cap \mathfrak{b})$. Then for any $\mathfrak{p}$-highest weight $w$ of $M$ there exists an embedding $\Delta(w) \hookrightarrow M$. Moreover, the cokernel of this embedding is again $\mathfrak{n}_{--}$ free and (its restriction) is a direct sum of self-dual projective modules in $\mathcal{O}(\mathfrak{a}, \mathfrak{a} \cap \mathfrak{b})$. 
Proof. Let $N \subset M$ be the submodule generated by the vectors of $M$ of weight $w \cdot 0$. From Lemma 2.8 we get that $N$ is a homomorphic image of $\hat{N}=\bigoplus_{i=1}^{k} \Delta(w)$, where $k$ is assumed to be minimal. The minimality of $k$, the self-duality, and the projectivity of both $M$ and $\Delta(w)$ as a-modules imply that the restriction of $\hat{N} \rightarrow N$ to the weight space $w \cdot 0$ is injective. Since both $M$ and $\Delta(w)$ are $\mathfrak{n}_{-}$-free, the constructed map must have trivial kernel and thus $N \cong \hat{N}$. Everything else is straightforward.

The following crucial fact describes the behavior of standard modules under translations through the wall.

Proposition 4.2. Let $w \in W(\mathfrak{p})$, and let $s$ be a simple reflection. Then the module $\theta_{s} \Delta(w) \in \mathcal{O}_{0}$ (or $\mathcal{O}_{0}^{\mathfrak{p}-\text { pres }}$ respectively) has a standard filtration of length two. Moreover, in both categories it fits into one of the following short exact sequences:

$$
\begin{array}{llllll}
\Delta(w) & \stackrel{a d j_{s}}{\hookrightarrow} & \theta_{s} \Delta(w) & \rightarrow & \Delta(w s) & \text { if } w s \in W(\mathfrak{p}) \text { and } w s>w, \\
\Delta(w s) & \hookrightarrow & \theta_{s} \Delta(w) & \stackrel{\text { adj }^{s}}{\rightarrow} & \Delta(w) & \text { if } w s \in W(\mathfrak{p}) \text { and } w s<w, \\
\Delta(w) & \stackrel{\text { adjs }}{\hookrightarrow} & \theta_{s} \Delta(w) & \rightarrow & \Delta(w) & \text { if } w s \notin W(\mathfrak{p}) .
\end{array}
$$

Proof. By Corollary 2.2 it is enough to prove the statement for $\mathcal{O}_{0}$. Our strategy is the following: first we use that $\theta_{s}$ doubles the number of simple Verma subquotients to prove the first part of the theorem. For the first two sequences we use the injectivity (surjectivity) of the adjunction morphism and the fact that $\theta_{s} \Delta(w)$ contains a unique submodule (has unique quotient), isomorphic to the standard modules given by the corresponding exact sequence above. For the last part we use the fact that $\Delta(w)$ is a direct sum of self-dual a-projectives.

Proposition 2.18 implies that $\Delta(w)$ is $\mathfrak{n}_{-}$-free and by Proposition 2.9 it is a direct sum of self-dual projective a-modules. Then the module $\theta_{s} \Delta(w)$ will also have both properties. Now let $x \in\{w, w s\}$ be minimal if $w s \in W(\mathfrak{p})$, and $x=w$ otherwise. Then every vector $v \in \theta_{s} \Delta(w)$ of weight $x \cdot 0$ satisfies $\mathfrak{n} v=0$ and hence there is an embedding of $\Delta(x)$ into $\theta_{s} \Delta(w)$ by Lemma 4.1. Let $K$ be the cokernel. By the same arguments there is an embedding of some $\Delta\left(x^{\prime}\right)$ into $K$, and the cokernel $K^{\prime}$ of the last embedding is again $\mathfrak{n}_{-}$-free. By [GJ, 3.6] we have $\left[\theta_{s} \Delta(w): L\left(w_{0}\right)\right]=2\left[\Delta(w): L\left(w_{0}\right)\right]$, hence $\left[K^{\prime}: L\left(w_{0}\right)\right]=0$ by Proposition 2.18 and therefore $K^{\prime}=0$. Furthermore, we get $K \cong \Delta(x s)$ comparing the highest weights and therefore $\theta_{s} \Delta(w)$ has a standard filtration of length two as indicated. It remains to prove that the adjunction morphisms fit into these sequences.

Assume now that $w s \in W(\mathfrak{p})$ and $w s>w$. Then the adjunction morphism $\Delta(w) \hookrightarrow \theta_{s} \Delta(w)$ is injective. As $w s>w$, we already saw that the standard subquotients of a standard filtration of $\theta_{s} \Delta(w)$ are $\Delta(w)$ and $\Delta(w s)$. But, since $w s>w$ we have $[\Delta(w s): S(w)]=0$ in $\mathcal{O}_{0}^{\mathfrak{p}-\text { pres }}$ and thus the kernel of $\theta_{s} \Delta(w) \rightarrow$ $\Delta(w s)$ coincides with the image of $\operatorname{adj}_{s}: \Delta(w) \hookrightarrow \theta_{s} \Delta(w)$. The case $w s \in W(\mathfrak{p})$, $w s<w$, follows by analogous arguments.

Finally, let us assume that $w s \notin W(\mathfrak{p})$. We saw already that both subquotients of a standard filtration of $\theta_{s} \Delta(w)$ are isomorphic to $\Delta(w)$ and that $\operatorname{adj}_{s}(\Delta(w))$ is injective. On the other hand, both $\Delta(w)$ and $\theta_{s} \Delta(w)$, considered as a-modules, are direct sums of self-dual projectives, and thus the image of the adjunction is 
an $\mathfrak{a}$-direct summand of $\Delta(w)$. This implies that the quotient is again a directsum of self-dual a-projectives and another application of Lemma 4.1 completes the proof.

Corollary 4.3. Both $\mathcal{F}(\Delta)$ and $\mathcal{F}(\nabla)$ are stable under translation functors.

Proof. For $\mathcal{F}(\Delta)$ the statement follows from Proposition 4.2, using exactness of translation functors. Since commutes with all $\theta_{s}$, the statement for $\mathcal{F}(\nabla)$ follows as well.

Corollary 4.4. Let $w \in W(\mathfrak{p})$ and let $s$ be a simple reflection such that $w s \in W(\mathfrak{p})$. Then, $\theta_{s} \Delta(w)$ is indecomposable and $\theta_{s} \Delta(w) \cong \theta_{s} \Delta(w s)$ (as modules both in $\mathcal{O}_{0}$ and $\mathcal{O}_{0}^{\mathfrak{p}-\text { pres }}$ ).

Proof. The first two sequences of Proposition 4.2 imply that the head of $\theta_{s} \Delta(w)$ is contained in $L(w) \oplus L(w s)$. On the other hand, the self-adjointness of $\theta_{s}$ implies that it only contains simple modules which are not annihilated by $\theta_{s}$, i.e., $\theta_{s} \Delta(w)$ has simple head and is therefore indecomposable. To prove the second statement let us assume that $w<w s$. From the self-adjointness of $\theta_{s}$ we get $\operatorname{Hom}_{\mathfrak{g}}\left(\theta_{s} \Delta(w), \theta_{s} \Delta(w s)\right) \cong \operatorname{Hom}_{\mathfrak{g}}\left(\Delta(w), \theta_{s}^{2} \Delta(w s)\right)$. Taking into account $\theta_{s}^{2} \cong$ $\theta_{s} \oplus \theta_{s}$ and Proposition [2.9] we get from Proposition 4.2 that

$$
\begin{aligned}
& \operatorname{dim} \operatorname{Hom}_{\mathfrak{g}}\left(\Delta(w), \theta_{s} \Delta(w s) \oplus \theta_{s} \Delta(w s)\right)=\operatorname{dim} \operatorname{Hom}_{\mathfrak{g}}\left(\Delta(w), \theta_{s} \Delta(w) \oplus \theta_{s} \Delta(w)\right) \\
& =2 \operatorname{dim} \operatorname{End}_{\mathfrak{a}}\left(P^{\mathfrak{a}}(w \cdot 0)\right) \\
& =2\left|W_{\mathfrak{p}}\right| \text {. }
\end{aligned}
$$

We already know that both $\theta_{s} \Delta(w)$ and $\theta_{s} \Delta(w s)$ have a simple top isomorphic to $L(w s)$. Hence $\left[\operatorname{rad} \theta_{s} \Delta(w s): L(w s)\right]=2\left|W_{\mathfrak{p}}\right|-1$ by Lemma 2.18 and therefore

$$
\operatorname{dim} \operatorname{Hom}_{\mathfrak{g}}\left(\theta_{s} \Delta(w), \operatorname{rad} \theta_{s} \Delta(w s)\right) \leq 2\left|W_{\mathfrak{p}}\right|-1 .
$$

Therefore, there exists an element in $\operatorname{Hom}_{\mathfrak{g}}\left(\theta_{s} \Delta(w), \theta_{s} \Delta(w s)\right)$, whose image is not contained in the radical of $\theta_{s} \Delta(w s)$. Since $\theta_{s} \Delta(w s)$ has a simple top, a comparison of characters shows that the last homomorphism must be an isomorphism.

\section{ShufFLing IN $\mathcal{O}_{0}^{\mathfrak{p}-\text { pres }}$}

5.1. Shuffling and coshuffling on $\mathcal{O}_{0}$ and their derived functors. Let $M$, $N \in \mathcal{O}_{0}$. For a simple reflection $s$ we denote by $K_{s} M$ and $C_{s} M$ the kernel of the adjunction morphism $M \stackrel{\operatorname{adj}_{s}}{\longrightarrow} \theta_{s} M$ and the cokernel of the adjunction morphism $\theta_{s} M \stackrel{\text { adj }^{s}}{\longrightarrow} M$ respectively. By functoriality of $\theta_{s}$, each $f \in \operatorname{Hom}_{\mathfrak{g}}(M, N)$ gives rise to unique morphisms $K_{s} f \in \operatorname{Hom}_{\mathfrak{g}}\left(K_{s} M, K_{s} N\right)$ and $C_{s} f \in \operatorname{Hom}_{\mathfrak{g}}\left(C_{s} M, C_{s} N\right)$ via restriction and factorization respectively. Let $K_{s}$ and $C_{s}$ respectively denote the resulting endofunctors on $\mathcal{O}_{0}$. By construction, $K_{s}$ is left exact and $C_{s}$ is right exact. The functor $C_{s}$ is called shuffling functor (with respect to $s$ ) and is studied in [Ir1]. We call $K_{s}$ coshuffling with respect to $s$. They are connected by the following.

Lemma 5.1. Let $s$ be a simple reflection. Then $\left(C_{s}, K_{s}\right)$ is an adjoint pair of functors. 
Proof. Let $M, N \in \mathcal{O}_{0}$. Applying $\operatorname{Hom}_{\mathfrak{g}}(-, N)$ and $\operatorname{Hom}_{\mathfrak{g}}\left(M,_{-}\right)$to the exact sequences

$$
M \stackrel{\operatorname{adj}_{s}}{\longrightarrow} \theta_{s} M \longrightarrow C_{s} M \rightarrow 0, \quad \text { and } \quad 0 \rightarrow K_{s} N \longrightarrow \theta_{s} N \stackrel{\operatorname{adj}^{s}}{\longrightarrow} N,
$$

respectively, gives the following diagram with exact rows:

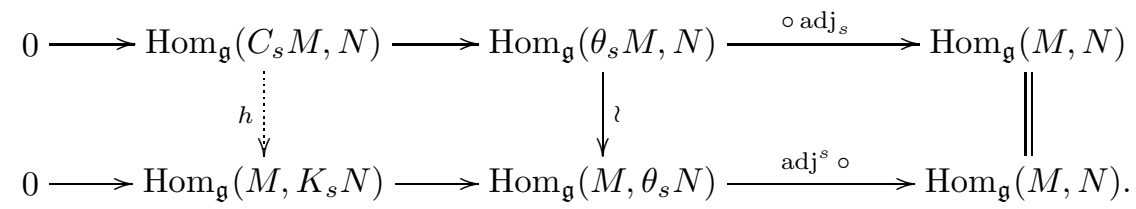

The self-adjointness of $\theta_{s}$ induces the middle vertical isomorphism. By the definition of the adjunction morphisms, the square on the right-hand side commutes. Hence, it induces a natural homomorphism $h$ as depicted in the diagram. By the five lemma, $h$ is an isomorphism and the lemma follows.

Moreover, the two functors are related by duality:

Lemma 5.2. For any simple reflection $s$, there is an isomorphism $K_{s} \cong C_{s}$ of endofunctors on $\mathcal{O}_{0}$

Proof. For $M \in \mathcal{O}_{0}$ we consider the exact sequence $M \stackrel{\operatorname{adj}_{s}}{\longrightarrow} \theta_{s} M \longrightarrow C_{s} M \rightarrow 0$. Dualizing gives an exact sequence $0 \rightarrow\left(C_{s} M\right) \longrightarrow\left(\theta_{s} M\right) \stackrel{\left(\operatorname{adj}_{s}\right)}{\longrightarrow} M^{*}$. The duality commutes with $\theta_{s}$ and the isomorphism $\varphi_{s}: \theta_{s} \sim \theta_{s}$ can be chosen such that the following diagram commutes:

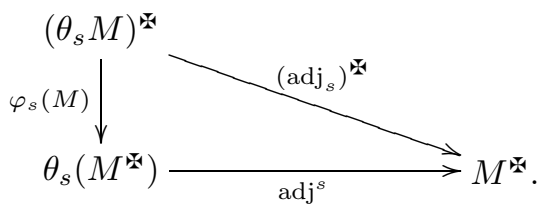

This defines an isomorphism $\left(C_{s} M\right)^{\text {N }} \cong K_{s}\left(M^{\text {w }}\right)$ which is canonical. Hence, we get an isomorphism of functors $C_{s} \cong K_{s}$. The statement of the lemma follows.

Let $\mathcal{L}^{i} C_{s}$ denote the $i$-th left derived functor of $C_{s}$. It is described by the following.

Proposition 5.3. For any simple reflection s the following holds:

(1) $\mathcal{L}^{i} C_{s} M(x)=0$ for $x \in W, i>0$.

(2) $C_{s}$ is exact when restricted to the full subcategory of modules having a Verma flag.

(3) $\mathcal{L}^{i} C_{s}= \begin{cases}\operatorname{ker}\left(\operatorname{adj}_{s}\right) & \text { if } i=1, \\ 0 & \text { if } i>1 .\end{cases}$

Remark 5.4. Dual statements hold for $\mathcal{R}_{i} K_{s}$, the $i$-th right derived functor of $K_{s}$.

Proof. Let $\mathcal{V}$ denote the full subcategory of $\mathcal{O}_{0}$ given by objects whose socle is a direct sum of the simple Verma module. In particular, Verma modules and all submodules of projective objects are objects of $\mathcal{V}$. Note that $\operatorname{adj}_{s}(M)$ is injective for any $M \in \mathcal{V}$. Hence, the Snake Lemma implies the exactness of $C_{s}$ when restricted to $\mathcal{V}$. This proves (1), (2) and the second part of (3). For $N \in \mathcal{O}_{0}$ arbitrary we 
choose a projective cover $P \stackrel{p}{\rightarrow} N$. Let $K$ denote the kernel of $p$. The Snake Lemma gives a short exact sequence

$$
0 \rightarrow \operatorname{ker}\left(\operatorname{adj}_{s}(M)\right) \longrightarrow C_{s} K \longrightarrow C_{s} P \longrightarrow C_{s} M \rightarrow 0 .
$$

The first part of the last statement follows and the proposition is proved.

Corollary 5.5. Let s be a simple reflection. When restricted to the full subcategory of modules with Verma flag, the adjunction morphism induces an isomorphism of functors $K_{s} C_{s} \cong \mathrm{ID}$.

Proof. We first claim that $K_{s} C_{s}$ is exact on modules with Verma flag. By the definition of $C_{s}$ the head of $C_{s} M$ for any $M \in \mathcal{O}_{0}$ is not annihilated by $\theta_{s}$. Hence, the adjunction morphism $\theta_{s}\left(C_{s} M\right) \stackrel{\text { adj }^{s}}{\rightarrow} C_{s} M$ is surjective. Now given a short exact sequence $S$ of modules with Verma flag we get by the previous Proposition 5.3 a short exact sequence $C_{s} S$ of modules, where adj ${ }^{s}$ is surjective. The Snake Lemma implies the claim.

Now let $x \in W$ such that $x s>x$. Then $K_{s} C_{s} M(x) \cong K_{s} M(x s) \cong M(x)$. On the other hand, there is a short exact sequence

$$
0 \rightarrow M(x s) \rightarrow M(x) \rightarrow Q \rightarrow 0,
$$

where $\theta_{s} Q=0$. Hence, by definition of $C_{s}$, the Snake Lemma gives a short exact sequence of the form $Q \hookrightarrow C_{s} M(x s) \rightarrow M(x s)$. Again by the Snake Lemma (and the definition of $\left.K_{s}\right)$, we get a short exact sequence of the form $K_{s} C_{s} M(x s) \hookrightarrow$ $M(x) \rightarrow Q$. This implies $K_{s} C_{s} M(x s) \cong M(x s)$. Therefore, we have an isomorphism $K_{s} C_{s} M(x) \cong M(x)$ for any $x \in W$, which is given by the adjunction morphism $K_{s} C_{s} \rightarrow \mathrm{ID}$. The full statement follows now easily by induction on the length of a Verma flag.

Corollary 5.6. When restricted to the full subcategory of projective modules, the adjunction morphism induces an isomorphism $K_{s} C_{s} \cong$ ID for any simple reflection $s$.

Proof. The claim follows from Corollary 5.5 because every projective has a Verma flag.

Let $\mathrm{D}^{b}\left(\mathcal{O}_{0}\right)$ denote the bounded derived category of $\mathcal{O}_{0}$. For any simple reflection $s$ we consider the endofunctors $\mathcal{L} C_{s}$ and $\mathcal{R}_{s} K_{s}$ of $\mathrm{D}^{b}\left(\mathcal{O}_{0}\right)$ induced from $C_{s}$ and $K_{s}$ respectively. The last corollary motivates the following.

Theorem 5.7. For any simple reflection $s$, the functor $\mathcal{L} C_{s}$ defines an autoequivalence of $\mathrm{D}^{b}\left(\mathcal{O}_{0}\right)$ with inverse functor $\mathcal{R} K_{s}$.

Proof. By Corollary 5.6 the adjunction induces a natural isomorphism $\mathcal{R} K_{s} \mathcal{L} C_{s} P$ $\cong P$ for any projective module $P \in \mathcal{O}_{0}$, since $C_{s} P$ is acyclic for $K_{s}$. On the other hand, any object of $\mathcal{D}^{b}\left(\mathcal{O}_{0}\right)$ is quasi-isomorphic to a finite complex including only projective objects. Hence $\mathcal{R} K_{s} \mathcal{L} C_{s} \cong$ ID. Dually, we have $\mathcal{L} C_{s} \mathcal{R} K_{s} I \cong I$ via the adjunction morphism for any injective object $I$. Now, any object of $\mathcal{D}^{b}\left(\mathcal{O}_{0}\right)$ is quasi-isomorphic to a finite complex including only injective objects. Hence $\mathcal{L} C_{s} \mathcal{R} K_{s} \cong \mathrm{ID}$.

Remark 5.8. The previous theorem follows directly by arguments used in Ric: We consider the following (co)complexes of endofunctors on $\mathcal{O}_{0}$ : 


$$
\begin{aligned}
& \left(\mathcal{C}_{s}\right)^{\bullet}: \quad \cdots \quad \longrightarrow \quad 0 \quad \longrightarrow \text { ID } \stackrel{\operatorname{adj}_{s}}{\longrightarrow} \theta_{s} \longrightarrow 0 \quad \longrightarrow \quad \longrightarrow \\
& \left(\mathcal{K}_{s}\right)_{\bullet}: \quad \quad \cdots \quad \longrightarrow 0 \longrightarrow \theta_{s} \stackrel{\text { adj }^{s}}{\longrightarrow} \text { ID } \longrightarrow 0 \longrightarrow \cdots \text {, }
\end{aligned}
$$

where $\theta_{s}$ is always concentrated in degree zero. Applying the (co)complex to objects of $\mathrm{D}^{b}\left(\mathcal{O}_{0}\right)$ and taking the total complex of the resulting double complex defines two endofunctors on $\mathrm{D}^{b}\left(\mathcal{O}_{0}\right)$ which coincide with $\mathcal{L} C_{s}$ and $\mathcal{R} K_{s}$, respectively. These are exactly the functors described in $[\mathrm{Ric}$ in the setup of reductive groups in positive characteristic. Rickard proves that such functors are inverse to each other and therefore define an auto-equivalence on the derived category corresponding to regular integral blocks. His arguments can be transfered mutatis mutandis to our situation, giving an alternative proof of Theorem 5.7

5.2. Shuffling and coshuffling on $\mathcal{O}_{0}^{\text {p-pres }}$ and their derived functors. Shuffling with respect to a simple reflection $s$ preserves the category $\mathcal{O}_{0}^{\mathfrak{p}-\text { pres }}$. This is clear in the picture of Harish-Chandra bimodules. To see this directly we first observe that if $M \in \mathcal{O}_{0}$ has a projective cover contained in $\operatorname{Add}\left(P_{\mathfrak{p}}\right)$, then so does $C_{s} M$. Indeed, a projective cover $P \stackrel{p}{\rightarrow} M$ gives a surjection $f: \theta_{s} P \stackrel{\text { can }}{\rightarrow}$ $C_{s} P \stackrel{C_{s} p}{\longrightarrow} C_{s} M$ and $\theta_{s}$ preserves $\mathcal{O}_{0}^{\mathfrak{p}-\text { pres }}$. We assume now that $M \in \mathcal{O}_{0}^{\mathfrak{p}-\text { pres }}$. Note that $\operatorname{ker}\left(C_{s} p\right)$ is a quotient of $C_{s}(\operatorname{ker} p)$, hence its projective cover is contained in $\operatorname{Add}\left(P_{\mathfrak{p}}\right)$.

Given a simple object $L=L(x) \in \mathcal{O}_{0}$ such that $x \notin W(\mathfrak{p})$, the short exact sequence $P \hookrightarrow \theta_{s} P \rightarrow C_{s} P$ implies $\operatorname{Ext}_{\mathcal{O}}^{1}\left(C_{s} P, L\right)=0$. Altogether, the short exact sequence $\operatorname{ker}\left(C_{s} p\right) \hookrightarrow C_{s} P \stackrel{C_{s} p}{\longrightarrow} C_{s} M$ implies $\operatorname{Ext}_{\mathcal{O}}^{1}\left(C_{s} M, L\right)=0$. Hence the outer terms in the following exact sequence

$$
\operatorname{Hom}_{\mathfrak{g}}\left(\theta_{s} P, L\right) \longrightarrow \operatorname{Hom}_{\mathfrak{g}}(\operatorname{ker} f, L) \longrightarrow \operatorname{Ext}_{\mathcal{O}}^{1}\left(C_{s} M, L\right)=0
$$

vanish, i.e., the projective cover of $\operatorname{ker} f$ is contained in $\operatorname{Add}\left(P_{\mathfrak{p}}\right)$. This shows that $C_{s} M \in \mathcal{O}_{0}^{\mathfrak{p}-\text { pres }}$, that is, $C_{s}$ preserves $\mathcal{O}_{0}^{\mathfrak{p}-\text { pres }}$.

Of course, coshuffling does not preserve $\mathcal{O}_{0}^{\mathfrak{p}-\text { pres }}$ in general. If we consider $\mathrm{D}^{b}\left(\mathcal{O}_{0}^{\mathfrak{p}-\text { pres }}\right)$, the bounded derived category of $\mathcal{O}_{0}^{\mathfrak{p}-\text { pres }}$, then we get the following analogue of Theorem 5.7

Theorem 5.9. The composition $\mathcal{R} \mathfrak{L} C_{s} \mathcal{L} \mathfrak{i}$ defines an auto-equivalence of the category $\mathrm{D}^{b}\left(\mathcal{O}_{0}^{\mathfrak{p}-\text { pres }}\right)$ with inverse $\mathcal{R} j \mathcal{R} K_{s} \mathcal{L}$ i.

Proof. First we consider the category $\mathrm{D}^{-}\left(\mathcal{O}_{0}^{\mathfrak{p}-\text { pres }}\right)$. Note that $\mathfrak{j} \mathfrak{i}=\mathrm{ID}$ on $\mathcal{O}_{0}^{\mathfrak{p}-\text { pres }}$ and $\mathfrak{i j}=$ ID when restricted to the subcategory of $\mathfrak{p}$-presentable modules in $\mathcal{O}_{0}$. Since $C_{s}$ preserves $\mathcal{O}_{0}^{\mathfrak{p}-\text { pres }}$, we have an adjoint pair of functors $(F, G)$, where $F=\mathfrak{j} C_{s} \mathfrak{i}$ and $G=\mathfrak{j} K_{s} \mathfrak{i}$. The adjunction morphism ID $\rightarrow G F$ defines an isomorphism for any projective object $P \in \mathcal{O}_{0}^{\mathfrak{p}-\text { pres }}$ (using the fact that $C_{s}$ preserves $\mathcal{O}_{0}^{\mathfrak{p}-\text { pres }}$ and Corollary [5.6). On the other hand, $P$ is acyclic for $F$ and $F(P)$ is acyclic for $G$ by definition of the functors. Therefore, the adjunction defines an isomorphism $\left(\mathcal{R} \mathfrak{j} \mathcal{R} K_{s} \mathcal{L} \mathfrak{i}\right)\left(\mathcal{R} j \mathcal{L} C_{s} \mathcal{L} \mathfrak{i}\right) \cong$ ID when restricted to projective modules (considered as objects in the derived category). Now, any object in $\mathcal{O}_{0}^{\mathfrak{p} \text {-pres }}$ can be replaced by a (possibly infinite) negative complex including only projectives. Hence $\left(\mathcal{R} \mathfrak{j} \mathcal{R} K_{s} \mathcal{L i}\right)\left(\mathcal{R} j \mathcal{L} C_{s} \mathcal{L i}\right) \cong \mathrm{ID}$ as endo-functors of $\mathrm{D}^{-}\left(\mathcal{O}_{0}^{\mathfrak{p}-\text { pres }}\right)$.

Consider now the category $\mathrm{D}^{+}\left(\mathcal{O}_{0}^{\mathfrak{p}-\text { pres }}\right)$. Recall that any injective object $I \in$ $\mathcal{O}_{0}^{\mathfrak{p}-\text { pres }}$ is a direct sum of projective-injective modules from $\mathcal{O}(\mathfrak{a}, \mathfrak{a} \cap \mathfrak{b})$ and that the adjunction morphism $\operatorname{adj}^{s}(I)$ is surjective for any simple reflection $s$. Hence $K_{s} I$ is 
$\mathfrak{p}$-presentable by Proposition 2.10. Therefore, the adjunction morphism $F G \rightarrow$ ID defines an isomorphism $j C_{s} \mathfrak{i j} K_{s} \mathfrak{i}(I)=\mathfrak{j} C_{s} K_{s} \mathfrak{i}(I) \cong I$, giving rise to the desired isomorphism of functors $\left(\mathcal{R} j \mathcal{L} C_{s} \mathcal{L i}\right)\left(\mathcal{R} \mathfrak{j} K_{s} \mathcal{L} \mathfrak{i}\right) \cong$ ID.

Using Proposition 5.3 and Remark 5.4 we obtain that $\mathcal{R j} \mathcal{L} C_{s} \mathcal{L i}$ and $\mathcal{R} j \mathcal{R} K_{s} \mathcal{L i}$ both preserve $\mathrm{D}^{b}\left(\mathcal{O}_{0}^{\mathfrak{p}-\text { pres }}\right)$. Hence the statement of the theorem follows now from what has been proved in the first two paragraphs and the fact that the category $\mathrm{D}^{b}\left(\mathcal{O}_{0}^{\mathfrak{p}-\text { pres }}\right)$ is the intersection of the categories $\mathrm{D}^{-}\left(\mathcal{O}_{0}^{\mathfrak{p}-\text { pres }}\right)$ and $\mathrm{D}^{+}\left(\mathcal{O}_{0}^{\mathfrak{p}-\text { pres }}\right)$.

5.3. Shuffled standard objects. Let $x \in W$ with a fixed reduced expression $x=s_{1} \cdot \ldots \cdot s_{r}$. We define $C_{s}=C_{s_{r}} \cdots C_{s_{1}}$ and $K_{x}=K_{s_{r}} \cdots K_{s_{1}}$. Note that $\left(C_{x}, K_{x^{-1}}\right)$ defines an adjoint pair of functors by Lemma 5.1. For $M \in \mathcal{O}_{0}$ we call $K_{x}(M)$ the $x$-coshuffle of $M$ and $C_{x}(M)$ the $x$-shuffle of $M$. So far it is not clear that these definitions do not depend on the chosen reduced expression. To see this we have to work in the setup of Harish-Chandra bimodules. Since we do not want to introduce too many new notations, we just sketch the proof. For details we refer to St1:

Lemma 5.10. Let $x \in W$ and $M \in \mathcal{O}_{0}$. Up to isomorphism, both $K_{x} M$ and $C_{x} M$ are independent of the reduced expression of $x$.

Sketch of the proof. For $n \in \mathbb{N}$ and $\lambda \in \mathfrak{h}^{*}$ we consider the thickened Verma modules

$$
M^{n}(\lambda)=\mathcal{U}(\mathfrak{g}) \otimes_{\mathcal{U}(\mathfrak{b})} S(\mathfrak{h}) /(\operatorname{ker} \lambda)^{n} .
$$

Note that $M^{1}(\lambda)=M(\lambda)$ is the usual Verma module. Let $\eta:{ }_{0}^{\infty} \mathbf{H}_{0}^{\infty} \longrightarrow{ }_{0}^{\infty} \mathbf{H}_{0}^{\infty}$ denote the endofunctor which is given by switching the left and right action of $\mathfrak{g}$. Since tensoring with finite dimensional left (or right) $\mathfrak{g}$-modules preserves $\mathbf{H}$, one can define translations through the wall. We fix a simple reflection $s$. Let $\theta_{s}^{l}$ and $\theta_{s}^{r}$ denote the translation through the $s$-wall from the left and right hand side respectively. Let $E^{l}$ (and $E^{r}$ respectively) be a finite dimensional $\mathcal{U}(\mathfrak{g})$-bimodule with trivial right (left) action. It is easy to see that there are isomorphisms $\eta(X \otimes$ $\left.E^{l}\right) \cong \eta(X) \otimes E^{r}$ and $\eta\left(X \otimes E^{r}\right) \cong \eta(X) \otimes E^{l}$ for any $X \in{ }_{0}^{\infty} \mathbf{H}_{0}^{\infty}$. This implies isomorphisms of functors (for details see [Ja, 6.33-6.35])

$$
\eta \theta_{s}^{r} \cong \theta_{s}^{l} \eta \quad \text { and } \quad \eta \theta_{s}^{l} \cong \theta_{s}^{r} \eta .
$$

Let $k_{s}^{r}$ denote the endofunctor on ${ }_{0}^{\infty} \mathbf{H}_{0}^{\infty}$ which is given by taking the kernel of the adjunction morphism r-adj $: \theta_{s}^{r} \rightarrow$ ID. Similarly, let $k_{s}^{l}$ denote the endofunctor given by taking the kernel of the adjunction morphism l-adj ${ }^{s}: \theta_{s}^{l} \rightarrow$ ID. The isomorphisms (5.1) imply $\eta k_{s}^{r} \cong k_{s}^{l} \eta$.

Now let $M, N \in \mathcal{O}_{0}^{\infty}$ (see section 3.1). Translation gives a natural isomorphism $\theta_{s}^{r} \mathcal{L}(M, N) \cong \mathcal{L}\left(\theta_{s} M, N\right)$ such that the following diagram with exact rows and induced isomorphism $q$ commutes:

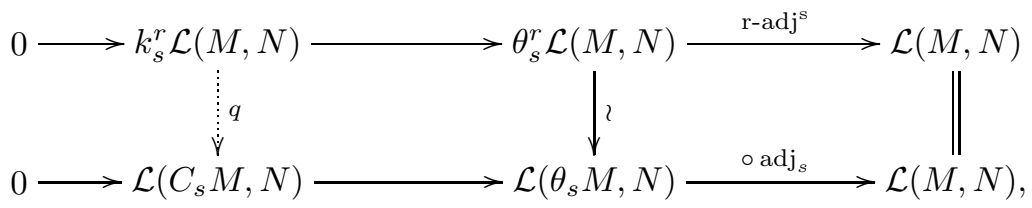


where $C_{s}$ denotes the corresponding shuffling functor in $\mathcal{O}_{0}^{\infty}$. Since the modules $M^{n}(x \cdot 0)$ behave similar to Verma modules under translation, we get $C_{s} M^{n}(x \cdot 0) \cong$ $M^{n}(x s \cdot 0)$ if $x s>x$.

On the other hand (see [So3]), any $X \in{ }_{0}^{\infty} \mathbf{H}_{0}^{\infty}$ is isomorphic to $\mathcal{L}\left(M^{n}(0), N\right)$ for some $n \in \mathbb{N}$ and $N \in \mathcal{O}_{0}^{\infty}$. Hence, with $M=M^{n}(0)$, the diagram (5.2) above implies $k_{s}^{r} X \cong \mathcal{L}\left(M^{n}(s \cdot 0), N\right)$ and inductively $k_{x}^{r} X=k_{s_{r}}^{r} \cdot \ldots \cdot k_{s_{1}}^{r} X \cong$ $\mathcal{L}\left(M^{n}(x \cdot 0), N\right)$ for any reduced expression $x=s_{1} \cdot \ldots \cdot s_{r}$ of $x$. In particular, it does not depend on the actual choice of the reduced expression.

A diagram, analogous to (5.2), provides an isomorphism,

$$
k_{s}^{l} X \cong k_{s}^{l} \mathcal{L}\left(M^{n}(0), N\right) \cong \mathcal{L}\left(M^{n}(0), K_{s} N\right),
$$

where $K_{s}$ denotes the coshuffling on $\mathcal{O}_{0}^{\infty}$. With the isomorphism of functors $\eta k_{s}^{r} \cong$ $k_{s}^{l} \eta$ we get that $k_{x}^{l} X=k_{s_{r}}^{l} \cdot \ldots \cdot k_{s_{1}}^{l} X$ does not depend on the choice of the reduced expression for $x$ and hence $k_{x}^{l} X \cong \mathcal{L}\left(M^{n}(0), K_{x} N\right)$ as well. Therefore, $K_{x} N$ does not depend (up to isomorphism) on the reduced expression of $x$ (by the equivalence of [So3]). The arguments for $C_{x} M$ are analogous.

Remark 5.11. One can show that $K_{x}$ and hence $C_{x}$ are independent of the reduced expression of $x$ even as functors (see [St1]).

For $x \in W(\mathfrak{p})$ and $y \in W$ we define $\Delta(x, y)=C_{y}(\Delta(x))$ (it is well defined by Lemma 5.10). In particular, $\Delta(x, e)=\Delta(x)$. To illustrate this definition we will give two detailed examples in Section 9 , For $w \in W$ we denote by $\bar{w}$ the longest element in the coset $W_{\mathfrak{p}} w$. The basic properties of $\Delta(x, y)$ are collected in the following statement:

Proposition 5.12. Let $x \in W(\mathfrak{p})$ and $y \in W$. In both $\mathcal{O}_{0}$ and $\mathcal{O}_{0}^{\mathfrak{p}-\text { pres }}$ the following holds:

(1) Let $s$ be a simple reflection such that $y s>y$. The adjunction morphism on $\Delta(x, y)$ is injective and fits into the following short exact sequence:

$$
0 \rightarrow \Delta(x, y) \stackrel{\operatorname{adj}_{s}}{\longrightarrow} \theta_{s} \Delta(x, y) \rightarrow \Delta(x, y s) \rightarrow 0 .
$$

(2) We have $[\Delta(x, y)]=[\Delta(x y)]$, i.e., the characters of the modules $\Delta(x, y)$ and $\Delta(x y)$ coincide.

(3) $\Delta(x, y)^{\text {w }} \cong \Delta\left(\overline{x w_{0}}, w_{0} y\right)$.

(4) $\Delta(x, y)$ is indecomposable.

(5) Let $s$ be a simple reflection such that $x s>x$ and $s y<y$. Then $\Delta(\overline{x s}, s y) \cong$ $\Delta(x, y)$.

(6) Let $s$ be a simple reflection. Then $\theta_{s} \Delta(x, y) \cong \theta_{s} \Delta(x, y s)$.

Proof. The proof is the same as the proof of corresponding statements for shuffled

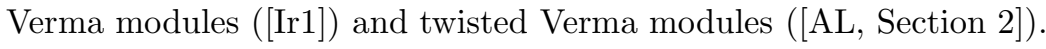

Remark 5.13. One can make a parallel with [AL, Section 2] and say that the modules $\{\Delta(x, y)\}$ are uniquely defined by properties described in Proposition 5.12 together with $\Delta(\bar{e}, e)=\Delta(\bar{e})$. Hence they define a family of "twisted standard modules" in $\mathcal{O}_{0}^{\mathfrak{p}-\text { pres }}$. One might expect that $\Delta(x, y)$ corresponds to $\mathcal{L}\left(\Delta\left(w_{0} \cdot 0\right)\right.$, $\left.\nabla\left(w_{0} x y \cdot 0\right)\right)$ as in the regular case. Comparing the characters, however, shows that this is not true. 
5.4. Shuffled filtrations of projective and tilting modules. In this section we start with an analogue of Irving's results from [Ir1, Section 4] for shuffled filtrations on projective modules. They are now easy corollaries of the results from the Subsections 5.1 and 5.4. For $M \in \mathcal{O}_{0}^{\mathfrak{p}-\text { pres }}$ and $y \in W$ we say that $M$ has a $y$-shuffled standard flag if there is a filtration of $M$, whose subquotients are isomorphic to modules $\Delta(t, y), t \in W(\mathfrak{p})$. Since shuffling preserves $\mathcal{O}_{0}^{\mathfrak{p}-\text { pres }}$, Corollary 2.2 ensures that we can consider $M$ and its filtration inside $\mathcal{O}_{0}^{\mathfrak{p}-\text { pres }}$. We denote by $\mathcal{F}(\Delta, y)$ the full subcategory of $\mathcal{O}_{0}^{\mathfrak{p}-\text { pres }}$, consisting of all modules, having a $y$-shuffled standard flag. We also note that $\mathcal{F}\left(\Delta, w_{0}\right)=\mathcal{F}(\nabla)$

Lemma 5.14. Assume that $M \in \mathcal{F}(\Delta, y)$ for some $y \in W$ and $s$ is a simple reflection such that $y s>y$. Then $C_{s} M \in \mathcal{F}(\Delta, y s)$.

Proof. From Subsection 5.1 and the condition $y s>y$ it follows that $C_{s}$ is exact on the category of all modules having a $y$-shuffled standard flag and the statement follows.

For $x \in W$ we denote by $W^{x}$ the subgroup of $W$, generated by all simple reflections $s$, satisfying $x s<x$.

Theorem 5.15. Let $x \in W(\mathfrak{p})$. For any $y \in W^{x}$ we have $P(x) \in \mathcal{F}(\Delta, y)$. In particular, $P\left(w_{0}\right) \in \mathcal{F}(\Delta, y)$ for all $y \in W$.

Proof. For a simple reflection $s \in W^{x}$ a comparison of characters yields $\theta_{s} P(x) \cong$ $P(x) \oplus P(x)$ and thus $C_{s} P(x) \cong P(x)$. Now the statement follows by induction in the length of $y$ using Lemma 5.14 .

Recall (see for example [FKM2, Section 6]) that tilting modules in $\mathcal{O}_{0}^{\mathfrak{p}-\text { pres }}$ are defined as self-dual modules having a standard filtration. It is well known that every tilting module is a direct sum of indecomposable tilting modules, and the latter ones bijectively correspond to simple modules and are therefore indexed by $w \in W(\mathfrak{p})$. For $w \in W(\mathfrak{p})$ we denote by $T(w)$ the indecomposable tilting module having a standard filtration starting with $\Delta(w)$ as a submodule. The next theorem is a generalization to $\mathcal{O}_{0}^{\mathfrak{p}-\text { pres }}$ of the main result of $\mathrm{Ma}$.

Theorem 5.16. Let $T$ be a tilting module. Then $T \in \mathcal{F}(\Delta, y)$ for all $y \in W$.

To prove the theorem we will need the following observations:

Lemma 5.17. Assume that $M \in \mathcal{F}(\Delta, y)$ for all $y \in W$. Then $\theta_{s} M \in \mathcal{F}(\Delta, y)$ for all $y \in W$ and for any simple reflection $s \in W$.

Proof. As $M$ has both a standard and a costandard filtration, it is a tilting module, and thus $\theta_{s} M$ is a tilting module as well by Corollary 4.3 In particular, the adjunction morphism $M \stackrel{\text { adj }_{s}}{\rightarrow} \theta_{s} M$ is injective and $C_{s} M \in \mathcal{F}(\Delta, y)$ for any $y \in W$ such that $y s<y$. Since $M \in \mathcal{F}(\Delta, y)$ for any $y \in W$, we get that $\theta_{s} M \in \mathcal{F}(\Delta, y)$ for any $y \in W, y s<y$. On the other hand, $\theta_{s} M$ is self-dual and thus if $\theta_{s} M \in \mathcal{F}(\Delta, y)$ for some $y \in W$, we have $\theta_{s} M \in \mathcal{F}\left(\Delta, w_{0} y\right)$. This implies that $\theta_{s} M \in \mathcal{F}(\Delta, y)$ for any $y \in W$ and completes the proof.

Lemma 5.18. There is an equivalence $\mathcal{F}(\Delta, x) \cong \mathcal{F}(\Delta, y)$ of categories for all $x, y \in W$. In particular, $\mathcal{F}(\Delta, y)$ is closed under taking direct summands for all $y \in W$. 
Proof. Without loss of generality, we can assume $x=y s$ for some simple reflection $s$ and $y s<y$. By the same arguments as in Corollary [5.5 one shows that the adjunction morphisms induce the isomorphism of functors $K_{s} C_{s} \cong$ ID $: \mathcal{F}(\Delta, y) \rightarrow$ $\mathcal{F}(\Delta, y)$ and $C_{s} K_{s} \cong \mathrm{ID}: \mathcal{F}(\Delta, y s) \rightarrow \mathcal{F}(\Delta, y s)$. Hence $K_{s}: \mathcal{F}(\Delta, y s) \rightarrow \mathcal{F}(\Delta, y)$ and $C_{s}: \mathcal{F}(\Delta, y) \rightarrow \mathcal{F}(\Delta, y s)$ are mutually inverse equivalences of categories. Now the second statement follows from the first one and Corollary 2.14

Proof of Theorem 5.16. We start with the module $\Delta\left(w_{0}\right)$. This module is a translation of a simple Verma module, hence it is self-dual and thus tilting. From the second part of Proposition 5.12 it follows that for every $y \in W$ there exists $x \in W(\mathfrak{p})$ such that $\Delta(x, y) \cong \Delta\left(w_{0}\right)$. Hence $\Delta\left(w_{0}\right) \in \mathcal{F}(\Delta, y)$ for any $y \in W$.

Translating $\Delta\left(w_{0}\right)$ produces new tilting modules and every indecomposable tilting module is a direct summand of some $E \otimes \Delta\left(w_{0}\right)$ with $E$ finite dimensional (see CI]). By Lemma 5.17, all $\mathcal{O}_{0}$-projections of $E \otimes \Delta\left(w_{0}\right)$ belong to $\mathcal{F}(\Delta, y)$ for any $y \in W$. Further, by Lemma 5.18 , every direct summand $M$ of $E \otimes \Delta\left(w_{0}\right)$ belongs to $\mathcal{F}(\Delta, y)$ for any $y \in W$, which completes the proof .

\section{Socles of STANDARD MOdules AND ENDOMORPHiSM RingS OF SHUFFLED STANDARD MODULES}

In this section we study the standard modules from $\mathcal{O}_{0}^{\mathfrak{p}-\text { pres }}$ in more detail. The main results of this section are the following statements.

Theorem 6.1. Let $w \in W(\mathfrak{p})$.

(1) The action of $Z(\mathfrak{g})$ on $\Delta(w)$ gives rise to a surjection $Z(\mathfrak{g}) \rightarrow \operatorname{End}_{\mathfrak{g}}(\Delta(w))$. Moreover, $\operatorname{End}_{\mathfrak{g}}(\Delta(w))$ does not depend on $w$.

(2) The module $\Delta(w)$ (considered as an object of both $\mathcal{O}_{0}^{\mathfrak{p}-\text { pres }}$ and $\mathcal{O}$ ) has simple socle.

Remark 6.2. From the proof of Theorem 6.1 and [So2, Endomorphismensatz] it follows that $\operatorname{End}_{\mathfrak{g}}(\Delta(w))$ is isomorphic to the coinvariant algebra, associated with $\mathfrak{a}$.

The first part of Theorem 6.1 can be generalized as follows:

Theorem 6.3. Let $x \in W(\mathfrak{p})$ and $y \in W$. Then the algebra $\operatorname{End}_{\mathfrak{g}}(\Delta(x, y))$ is commutative, moreover, the action of $Z(\mathfrak{g})$ on $\Delta(x, y)$ gives rise to a surjection $Z(\mathfrak{g}) \rightarrow \operatorname{End}_{\mathfrak{g}}(\Delta(x, y))$

To prove this theorem we have to recall the notion of the $\mathfrak{p}$-Harish-Chandra homomorphism from [DFO]. For the algebras $U(\mathfrak{g})$ and $U(\mathfrak{a} \oplus \mathfrak{h} \mathfrak{a})$ we let $U(\mathfrak{g})_{0}$ and $U\left(\mathfrak{a} \oplus \mathfrak{h}_{\mathfrak{a}}^{\perp}\right)_{0}$ be the centralizers of $\mathfrak{h}$ in $U(\mathfrak{g})$ and $U\left(\mathfrak{a} \oplus \mathfrak{h}_{\mathfrak{a}}^{\perp}\right)$ respectively. Set $L(\mathfrak{g})=U(\mathfrak{g})_{0} \cap U(\mathfrak{g}) \mathfrak{n}$ and one easily computes that $U(\mathfrak{g})_{0} \cong L(\mathfrak{g}) \oplus U(\mathfrak{a}+\mathfrak{h})$. The projection $\varphi_{\mathfrak{p}}$ onto the second summand $U(\mathfrak{a}+\mathfrak{h}) \cong U(\mathfrak{a}) \otimes_{\mathbb{C}} U\left(\mathfrak{h} \mathfrak{a}^{\perp}\right)$ is called the $\mathfrak{p}$ Harish-Chandra homomorphism. It is easy to see that $\varphi_{\mathfrak{p}}(Z(\mathfrak{g}))=Z(\mathfrak{a}) \otimes_{\mathbb{C}} U(\mathfrak{h} \mathfrak{a})$. A crucial property of $\varphi_{\mathfrak{p}}$ is the following: Let $M$ be a $\mathfrak{g}$-module and $v \in M$. If $\mathfrak{n} v=0$, then $z v=\varphi_{\mathfrak{p}}(z) v$ for all $z \in Z(\mathfrak{g})$. In other words, the action of $Z(\mathfrak{g})$ on weight elements annihilated by $\mathfrak{n}$ is determined by the action of $Z(\mathfrak{a})$ and $U(\mathfrak{h} \mathfrak{a})$.

Fix $i \in\left\{0,1, \ldots, l\left(w_{0}^{\mathfrak{p}}\right)\right\}$ and $w \in W(\mathfrak{p})$, and denote by $S(w, i)$ the set of all $w^{\prime} \in W_{\mathfrak{p}} w$ such that $l(w)-l\left(w_{0}^{\mathfrak{p}}\right) \leq l\left(w^{\prime}\right) \leq l(w)-l\left(w_{0}^{\mathfrak{p}}\right)+i$. Let $N_{i}^{w}$ be the submodule of $\Delta(w)$ which occurs as a submodule in a Verma filtration of $\Delta(w)$ such that $\left[N_{i}^{w}\right]=\sum_{w^{\prime} \in S(w, i)}\left[M\left(w^{\prime}\right)\right]$. It is easy to see that $N_{i}^{w}$ is well defined. Set $K_{i}^{w}=\left\{v \in \Delta(w): \mathfrak{m}_{0}^{i} v=0\right\}$. 
Lemma 6.4. Let $w \in W(\mathfrak{p})$. Then $N_{i}^{w}=K_{i}^{w}$ for any $i \in\left\{0,1, \ldots, l\left(w_{0}^{\mathfrak{p}}\right)\right\}$

Proof. Since $\Delta(w) \cong U(\mathfrak{g}) \otimes_{U(\mathfrak{p})} P^{\mathfrak{a}}(w \cdot 0)$ (see Proposition 2.9) and the action of $Z(\mathfrak{g})$ commutes with the parabolic induction, the statement reduces to the corresponding statement for $P^{\mathfrak{a}}(w \cdot 0)$. The latter one is a weight module and $\mathfrak{n} P^{\mathfrak{a}}(w \cdot 0)=0$, hence the $\mathfrak{p}$-Harish-Chandra homomorphism reduces the action of $Z(\mathfrak{g})$ on $P^{\mathfrak{a}}(w \cdot 0)$ to that of $Z(\mathfrak{a})$. Now the statement follows from Ba Section 2.6].

Proof of Theorem 6.1. Since $\Delta(w)$ is a quotient of $P(w)$, it has a simple top, namely $S(w)$. As $[\bar{\Delta}(w): S(w)]=1$ by the definition of proper standard modules, it follows from Proposition 2.18 that $[\Delta(w): S(w)]=\left|W_{\mathfrak{p}}\right|$. Hence, $\operatorname{dim} \operatorname{End}_{\mathfrak{g}}(\Delta(w)) \leq\left|W_{\mathfrak{p}}\right|$. On the other hand, the parabolic induction gives an injection

$$
\operatorname{End}_{\mathfrak{a}}\left(P^{\mathfrak{a}}(w \cdot 0)\right) \hookrightarrow \operatorname{End}_{\mathfrak{g}}(\Delta(w)) .
$$

By [So2, Endomorphismensatz], the algebra $\operatorname{End}_{\mathfrak{a}}\left(P^{\mathfrak{a}}(w \cdot 0)\right)$ is commutative, has dimension $\left|W_{\mathfrak{p}}\right|$, and the action of $Z(\mathfrak{a})$ on $P^{\mathfrak{a}}(w \cdot 0)$ gives rise to a surjection onto $\operatorname{End}_{\mathfrak{a}}\left(P^{\mathfrak{a}}(w \cdot 0)\right)$. As $\mathfrak{n} P^{\mathfrak{a}}(w \cdot 0)=0$, the first statement now follows by applying the $\mathfrak{p}$-Harish-Chandra homomorphism.

To prove the second part we use Lemma 6.4 and get that the socle of $\Delta(w)$ coincides with the socle of $\bar{\Delta}(w)$. The latter one is known to have a simple socle in $\mathcal{O}_{0}^{\mathfrak{p}-\text { pres }}$ (see for example [FKM2]).

Remark 6.5. Unlike the socle of the standard modules, the socle of $\Delta(x, y)$ is not simple in general and is quite difficult to describe.

Proof of Theorem 6.3. Since $\Delta(x, y)$ is obtained from $\Delta(x, e)=\Delta(x)$ via shuffling, which is an equivalence of appropriate categories (see Lemma 5.18), it follows that $\operatorname{End}_{\mathfrak{g}}(\Delta(x, y)) \cong \operatorname{End}_{\mathfrak{g}}(\Delta(x))$; in particular, $\operatorname{End}_{\mathfrak{g}}(\Delta(x, y)) \cong \operatorname{End}_{\mathfrak{g}}\left(\Delta\left(x^{\prime}, y^{\prime}\right)\right)$ for all $x^{\prime} \in W(\mathfrak{p})$ and $y^{\prime} \in W$. Thus Theorem 6.1 implies that $\operatorname{End}_{\mathfrak{g}}(\Delta(x, y))$ is a commutative algebra of dimension $\left|W_{\mathfrak{p}}\right|$. To complete the proof it is enough to show that the image of $Z(\mathfrak{g})$ in $\operatorname{End}_{\mathfrak{g}}(\Delta(x, y))$ with respect to the natural action has dimension at least $\left|W_{\mathfrak{p}}\right|$.

The modules $\Delta(x, e)=\Delta(x)$ are direct sums of self-dual projective modules in $\mathcal{O}(\mathfrak{a}, \mathfrak{b} \cap \mathfrak{a})$ by Proposition 2.9, hence so is the module $\Delta(x, y)$ by the definition of $C_{x}$ and the injectivity of $\operatorname{adj}_{s}(\Delta(x, t))$ for $t s>t$. Let $\lambda^{\perp} \in\left(\mathfrak{h}_{\mathfrak{a}}^{\perp}\right)^{*}$ be maximal such that

$$
\Delta(x, y)_{\lambda^{\perp}}=\left\{v \in \Delta(x, y): h v=\lambda^{\perp}(h) v \text { for all } h \in \mathfrak{h}_{\mathfrak{a}}^{\perp}\right\} \neq 0 .
$$

As $\left[\mathfrak{a}, \mathfrak{h}_{\mathfrak{a}}^{\perp}\right]=0$, the $\mathfrak{a}$-module $\Delta(x, y)_{\lambda^{\perp}}$ is a direct summand of $\Delta(x, y)$, and therefore a direct sum of self-dual projective modules in $\mathcal{O}(\mathfrak{a}, \mathfrak{b} \cap \mathfrak{a})$. The maximality of $\lambda^{\perp}$ implies $\mathfrak{n} \Delta(x, y)_{\lambda^{\perp}}=0$ and hence the action of $Z(\mathfrak{g})$ on $\Delta(x, y)_{\lambda^{\perp}}$ reduces to the action of $Z(\mathfrak{a})$ via the $\mathfrak{p}$-Harish-Chandra homomorphism. From So2, Endomorphismensatz] it follows that the image of this action in $\operatorname{End}_{\mathfrak{a}}\left(\Delta(x, y)_{\lambda^{\perp}}\right)$ and hence the image of $Z(\mathfrak{g})$ in $\operatorname{End}_{\mathfrak{g}}(\Delta(x, y))$ has dimension at least $\left|W_{\mathfrak{p}}\right|$. This completes the proof.

\section{A Categorification of a Hecke module via $\mathcal{O}_{0}^{\mathfrak{p}-\text { pres }}$}

7.1. Graded lifts of standard objects in $\mathcal{O}_{0}^{\mathfrak{p}-\text { pres }}$. Recall (from Section 2.2) the equivalence of categories between $\mathcal{O}_{0}$ and the category of finitely generated (right) modules over the endomorphism ring $A$ of a minimal projective generator $P$. We 
denote the latter category by mof $-A$. In [BGS] and [St3] it is explained how the algebra $A$ can be equipped with a $\mathbb{Z}$-grading such that it becomes a positively graded Koszul algebra. In the following, we fix this grading on $A$ and denote by gmof $-A$ the category of finitely generated graded right $A$-modules. Note that graded in what follows always means $\mathbb{Z}$-graded. Let $\mathrm{f}:$ gmof $-A \longrightarrow$ mof $-A$ denote the functor which forgets the grading. Let $i \in \mathbb{Z}$. For a graded $A$-module $M=\bigoplus_{n \in \mathbb{Z}} M_{n}$ we denote by $M\langle i\rangle$ the shifted module with respect to its grading; i.e., $\mathrm{f}(M\langle i\rangle) \cong \mathrm{f}(M)$ and $(M\langle i\rangle)_{n}=M_{n-i}$. We say $M \in \mathcal{O}_{0}$ is gradable, if there exists $\tilde{M} \in$ gmof $-A$ such that $\mathrm{f}(\tilde{M}) \cong \operatorname{Hom}_{\mathfrak{g}}(P, M)$ as $A$-modules. In this case we call $\tilde{M}$, by abuse of language, a lift of $M$. In [St3], it is shown that all projective and injective objects, all simple objects and the (dual) Verma modules are gradable. Moreover,

Lemma 7.1 ([St3, Lemma 2.5] or [BGS, Lemma 2.5.3]). A lift of an indecomposable gradable object in $\mathcal{O}_{0}$ is unique up to isomorphism and grading shift.

The lifts of the simple modules are all concentrated in one single degree. For any $x \in W$ we denote the graded lifts of $P(x), M(x)$ and $L(x)$ with the (up to isomorphism) characterizing property that their heads are all concentrated in degree zero by $\tilde{P}(x), \tilde{M}(x)$ and $\tilde{L}(x)$, respectively.

For any simple reflection $s$, there is an exact $\mathbb{Z}$-functor $\tilde{\theta}_{s}:$ gmof $-A \longrightarrow$ gmof $-A$ (in the sense of [A.JS, Appendix E]) which becomes translation through the $s$-wall on $\mathcal{O}_{0}$ after forgetting the grading and using the equivalence of categories $\mathcal{O}_{0} \tilde{\rightarrow}$ gmof $-A$. This functor is self-adjoint. The graded adjunction morphisms $\operatorname{Adj}_{s}(M): M \longrightarrow \tilde{\theta}_{s} M$ and $\operatorname{Adj}^{s}(M): \tilde{\theta}_{s} M \longrightarrow M$ turn out to be graded morphisms, homogeneous of degree 1 , for any $M \in$ gmof $-A$. The graded lifts of Verma modules fit into short exact sequences

$$
\begin{aligned}
& 0 \rightarrow \tilde{M}(x)\langle 1\rangle \stackrel{\operatorname{Adj}_{s}}{\longrightarrow} \tilde{\theta}_{s} M(x) \longrightarrow \tilde{M}(x s) \rightarrow 0 \quad \text { if } x s>x, \\
& 0 \rightarrow \tilde{M}(x s) \longrightarrow \tilde{\theta}_{s} M(x) \stackrel{\mathrm{Adj}^{s}}{\longrightarrow} \tilde{M}(x)\langle 1\rangle \rightarrow 0 \quad \text { if } x s<x,
\end{aligned}
$$

for any $x \in W$ and simple reflection $s$ (see [St3, Theorems 4.6 and 6.3].) Our first step is to prove that the standard objects in $\mathcal{O}_{0}^{\mathfrak{p}-\text { pres }}$ are gradable as objects of $\mathcal{O}_{0}$ :

Lemma 7.2. Let $x \in W(\mathfrak{p})$. Then $\Delta(x) \in \mathcal{O}_{0}$ is gradable. Moreover, a graded lift is unique up to isomorphism and shift of the grading.

Proof. Let $w=w_{0}^{\mathfrak{p}} \in W(\mathfrak{p})$ be minimal. Then $\Delta(w)=P(w)$ and therefore gradable. Assume, $\Delta(w)$ is gradable with lift $\tilde{\Delta}(w)$ and let $s$ be a simple reflection such that $w s>w$ and $w s \in W(\mathfrak{p})$. By Proposition 4.2, there is a short exact sequence $\Delta(w) \stackrel{a d j_{s}}{\hookrightarrow} \theta_{s} \Delta(w) \rightarrow \Delta(w s)$. It follows that the cokernel of the graded adjunction morphism $\operatorname{Adj}_{s}: \tilde{\Delta}(w)\langle 1\rangle \rightarrow \tilde{\theta}_{s} \tilde{\Delta}(w)$ defines a graded lift of $\Delta(w s)$. By induction on the length of $w$ we get the gradability of all $\Delta(w), w \in W(\mathfrak{p})$. Since all standard objects have a simple head, they are indecomposable. Hence their graded lifts are unique up to isomorphism and shift of the grading by Lemma 7.1 .

Convention. From now on we fix for any $w \in W(\mathfrak{p})$ a graded lift $\tilde{\Delta}(w)$ of $\Delta(w)$ which is characterized up to isomorphism by requiring that its head is concentrated in degree zero. Note, that this is compatible with our conventions for the lifts of projectives. 
Proposition 7.3. Let $s$ be a simple reflection. For any $w \in W(\mathfrak{p})$ the graded modules $\tilde{\Delta}(w)$ fit into short exact sequences of the form

$$
\begin{array}{llllll}
\tilde{\Delta}(w)\langle 1\rangle & \stackrel{\operatorname{Adj}_{s}}{\hookrightarrow} & \tilde{\theta}_{s} \tilde{\Delta}(w) & \rightarrow & \tilde{\Delta}(w s) & \text { if } w s \in W(\mathfrak{p}) \text { and } w s>w, \\
\tilde{\Delta}(w s) & \hookrightarrow & \tilde{\theta}_{s} \tilde{\Delta}(w) & \stackrel{\operatorname{Adj}^{s}}{\rightarrow} & \tilde{\Delta}(w)\langle-1\rangle & \text { if } w s \in W(\mathfrak{p}) \text { and } w s<w, \\
\tilde{\Delta}(w)\langle 1\rangle & \stackrel{\operatorname{Adj}_{s}}{\hookrightarrow} & \tilde{\theta}_{s} \tilde{\Delta}(w) & \rightarrow & \tilde{\Delta}(w)\langle-1\rangle & \text { if } w s \notin W(\mathfrak{p}) .
\end{array}
$$

Proof. Note that, if we forget the grading, we get exactly the short exact sequences of Proposition 4.2. We start with the case where $w s \in W(\mathfrak{p})$ and $w s>w$. Since the adjunction morphisms are all homogeneous of degree 1 , we get, by the definition of $\tilde{\Delta}(w s)$, a short exact sequence of graded $A$-modules of the form

$$
0 \rightarrow \tilde{\Delta}(w)\langle 1\rangle \stackrel{\operatorname{Adj}_{s}}{\longrightarrow} \tilde{\theta}_{s} \tilde{\Delta}(w) \longrightarrow \tilde{\Delta}(w s)\langle j\rangle \rightarrow 0
$$

for some $j \in \mathbb{Z}$. If $w=x \in W(\mathfrak{p})$ is minimal, then $\tilde{\Delta}(x) \cong \tilde{P}(x)$, hence there is a surjection $p_{x}: \tilde{\Delta}(x) \rightarrow \tilde{M}(x)$ of graded $A$-modules (St3], Corollary 8.2 and Theorem 8.5]). By functoriality, using the second sequence of (7.1), we get the following diagram with exact rows and commuting left square:

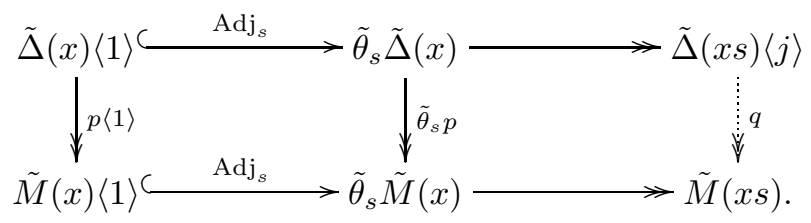

The commutativity gives rise to a graded surjection $q$ as pictured such that the whole diagram commutes. This forces $j=0$ by our conventions. Since we constructed a surjection $\tilde{\Delta}(x s) \rightarrow \tilde{M}(x s)$ of graded $A$-modules, we can apply induction on the length of $w$. This shows the existence of a short exact sequence of graded $A$-modules as claimed for any $w \in W(\mathfrak{p})$ such that $w s>w$.

Now let $w s \in W(\mathfrak{p})$ and $w s<w$. By Proposition 4.2 and Lemma 7.2 we have a short exact sequence of the form

$$
0 \rightarrow \tilde{\Delta}(w s)\langle j\rangle \longrightarrow \tilde{\theta}_{s} \tilde{\Delta}(w) \stackrel{\operatorname{Adj}^{s}}{\longrightarrow} \tilde{\Delta}(w)\langle-1\rangle \rightarrow 0
$$

for some $j \in \mathbb{Z}$. Let $p_{w}: \tilde{\Delta}(w) \rightarrow \tilde{M}(w)$ be a graded surjection. By functoriality we get the following commuting diagram with exact rows and induced surjection $q$ :

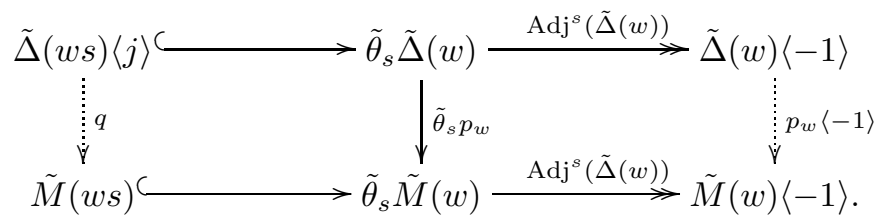

Therefore, the head of $\tilde{\Delta}(w s)\langle j\rangle$ has to be concentrated in degree zero, i.e., $j=0$. Hence (7.2) becomes the claimed sequence in the case $w, w s \in W(\mathfrak{p})$ and $w s<w$.

Now let $w \in W(\mathfrak{p})$ and $w s \notin W(\mathfrak{p})$. This implies, in particular, $w>w s$. We take again $p_{w}: \tilde{\Delta}(w) \rightarrow \tilde{M}(w)$, a surjection of graded $A$-modules. It gives a commutative 
diagram

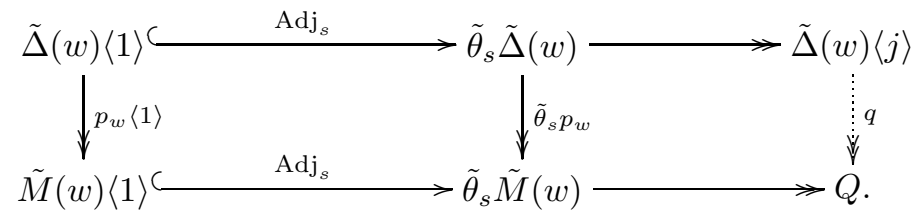

The first row is a short exact sequence of graded modules for some $j \in \mathbb{Z}$ by Proposition 4.2, and $Q$ is defined to make the lower row exact. The square on the left-hand side commutes by functoriality and induces therefore a surjection $q$ such that the whole diagram commutes. This implies that the head of $Q$ is isomorphic to $\tilde{L}(w)\langle j\rangle$. On the other hand, the adjunction morphism gives a surjection $\tilde{\theta}_{s} \tilde{M}(w) \rightarrow \tilde{M}(w)\langle-1\rangle$, hence the head of $Q$ is concentrated in degree -1 . This implies $j=-1$ and completes the proof.

Corollary 7.4. Let $w \in W(\mathfrak{p})$. If $s$ is a simple reflection such that $w s \in W(\mathfrak{p})$ and $w s>w$, then $\tilde{\theta}_{s} \tilde{\Delta}(w) \cong \tilde{\theta}_{s} \tilde{\Delta}(w s)\langle 1\rangle$.

Proof. By Corollary 4.4 there is an isomorphism $\theta_{s} \Delta(w) \cong \theta_{s} \Delta(w s)$, and these modules are indecomposable. Hence, the graded lifts are unique up to isomorphism and shift of the grading by Lemma 7.1. Therefore the existence of the first two exact sequences of Proposition 7.3 immediately implies $\tilde{\theta}_{s} \tilde{\Delta}(w) \cong \tilde{\theta}_{s} \tilde{\Delta}(w s)\langle 1\rangle$ if $w s>w$.

7.2. The parabolic Hecke module $\mathcal{M}$. Let us now consider the full abelian subcategory of gmof $-A$ generated by the projective modules $\tilde{P}(w)\langle j\rangle$ with $w \in$ $W(\mathfrak{p})$ and $j \in \mathbb{Z}$. This is a module category which is equivalent to gmof $-B$, where $B=\operatorname{End}_{\mathfrak{g}}\left(\bigoplus_{w \in W(\mathfrak{p})} P(w)\right)$ considered as a graded subalgebra of $A$.

Let $\mathbb{Z}\left[v, v^{-1}\right]$ denote the ring of Laurent polynomials in $v$ with coefficients in $\mathbb{Z}$. We consider the Weyl group $W$ as a Coxeter system, where the simple reflections form a set of generators $S \subset W$. Let $\mathcal{H}=\mathcal{H}(W, S)$ denote the corresponding Hecke algebra, which is by definition the free $\mathbb{Z}\left[v, v^{-1}\right]$-module with basis $\left\{H_{x} \mid x \in W\right\}$ and relations

$$
H_{x} H_{y}=H_{x y} \quad \text { if } l(x)+l(y)=l(x y), \text { and } \quad H_{s}^{2}=H_{e}+\left(v^{-1}-v\right) H_{s} \quad \text { for } s \in S .
$$

(compare [Bo $)$. Note that $\mathcal{H}$ is generated as an algebra over $\mathbb{Z}\left[v, v^{-1}\right]$ by $\left\{H_{s} \mid s \in\right.$ $S\}$; or (which will turn out to be more convenient) by the set $\left\{C_{s}=H_{s}+v H_{e} \mid s \in\right.$ $S\}$. Let $S_{\mathfrak{p}} \subset S$ be the subset generating $W_{\mathfrak{p}}$. Let $W^{\mathfrak{p}}$ denote the set of distinguished coset representatives of minimal length of $W_{\mathfrak{p}} \backslash W$. Set $\mathcal{H}_{\mathfrak{p}}=\mathcal{H}\left(W_{\mathfrak{p}}, S_{\mathfrak{p}}\right)$. Note that $\mathcal{H}$ has an obvious left $\mathcal{H}_{\mathfrak{p}}$-module structure via restriction. The natural $\mathbb{Z}\left[v, v^{-1}\right]$ bimodule structure on $\mathbb{Z}\left[v, v^{-1}\right]$ can be extended to an $\mathcal{H}_{\mathfrak{p}}$-module structure by letting $H_{s}$ (where $s \in S_{\mathfrak{p}}$ ) act via multiplication with $v^{-1}$. This bimodule structure defines the following $\left(\mathcal{H}_{\mathfrak{p}}, \mathcal{H}\right)$-bimodule

$$
\mathcal{M}_{\mathfrak{p}}:=\mathbb{Z}\left[v, v^{-1}\right] \otimes_{\mathcal{H}_{\mathfrak{p}}} \mathcal{H} .
$$

The bimodule $\mathcal{M}_{\mathfrak{p}}$ is called parabolic Hecke module. It is described in De and $[\mathrm{KL}]$, but we follow the exposition of [So1]. Considered as a left $\mathbb{Z}\left[v, v^{-1}\right]$-module, $\mathcal{M}_{\mathfrak{p}}$ is free with basis $\left\{M_{x}=1 \otimes H_{x} \mid x \in W^{\mathfrak{p}}\right\}$. The action of $\mathcal{H}$ is given by the following formulas. 
Lemma 7.5 (see [So1, Section 3]). Let $x \in W^{\mathfrak{p}}$ and $s \in S$. Then

$$
M_{x} C_{s}= \begin{cases}M_{x s}+v M_{x} & \text { if } x s \in W^{\mathfrak{p}} \text { and } x s>x, \\ M_{x s}+v^{-1} M_{x} & \text { if } x s \in W^{\mathfrak{p}} \text { and } x s<x, \\ \left(v+v^{-1}\right) M_{x} & \text { if } x s \notin W^{\mathfrak{p}} .\end{cases}
$$

7.3. The combinatorial description. We still fix $S_{\mathfrak{p}} \subseteq S$. The multiplication defines a bijection $W_{\mathfrak{p}} \times W^{\mathfrak{p}} \stackrel{1: 1}{\longleftrightarrow} W$ and we have $l(x)+l(y)=l(x y)$ for any $x \in W_{\mathfrak{p}}$ and $y \in W^{\mathfrak{p}}$ (see [Hu, 1.10]). On the other hand, it is easy to see that there is a bijection $W^{\mathfrak{p}} \stackrel{1: 1}{\longleftrightarrow} W(\mathfrak{p})$ given by $x \mapsto w_{0}^{\mathfrak{p}} x$. This implies that for any $s \in S$ and $x \in W^{\mathfrak{p}}$ we have $x s \in W^{\mathfrak{p}}$ if and only if $w_{0}^{\mathfrak{p}} x s \in W(\mathfrak{p})$. In the case, where $x s \in W^{\mathfrak{p}}$ we have $x s>x$ (or $x s<x$ ) if and only if $w_{0}^{\mathfrak{p}} x s>x$ (or $w_{0}^{\mathfrak{p}} x s<w_{0}^{\mathfrak{p}} x$ respectively).

The Grothendieck group [gmof $-B]$ becomes a $\mathbb{Z}\left[v, v^{-1}\right]$-module via $v^{i}[M]=$ $[M\langle i\rangle]$ for any $M \in$ gmof $-B$. The connection with the corresponding Hecke module $\mathcal{M}_{\mathfrak{p}}$ is given by the following lemma.

Lemma 7.6. There is an injective homomorphism of $\mathbb{Z}\left[v, v^{-1}\right]$-modules

$$
\begin{aligned}
\Phi_{\mathfrak{p}}: \mathcal{M}_{\mathfrak{p}} & \longrightarrow[\text { gmof }-B] \\
v^{i} H_{x} & \longmapsto\left[\tilde{\Delta}\left(w_{o}^{\mathfrak{p}} x\right)\langle i\rangle\right] .
\end{aligned}
$$

Proof. The map is well defined and injective, since $\left\{v^{i} H_{x} \mid x \in W^{\mathfrak{p}}, i \in \mathbb{Z}\right\}$ forms a $\mathbb{Z}$-basis of $\mathcal{M}_{\mathfrak{p}}$ and $\left[\tilde{\Delta}\left(w_{0}^{\mathfrak{p}} x\right)\langle i\rangle\right], x \in W^{\mathfrak{p}}, i \in \mathbb{Z}$ are linearly independent (over $\mathbb{Z}$ ) in [gmof $-B]$. By definition, the map $\Phi_{\mathfrak{p}}$ is compatible with the action of $\mathbb{Z}\left[v, v^{-1}\right]$.

Theorem 7.7. For any simple reflection $s$ the following diagram commutes:

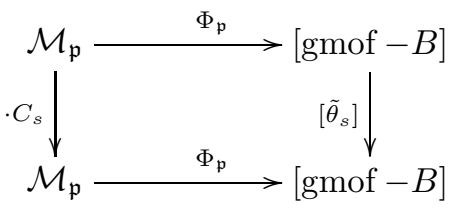

Proof. The proof is given by easy direct calculations. Let $i \in \mathbb{Z}$ and $x \in W^{\mathfrak{p}}$. Consider first the case $x s \in W^{\mathfrak{p}}$ and $x s>x$ (hence also $w_{0}^{\mathfrak{p}} x s>w_{0}^{\mathfrak{p}} x$ ). Then $\left[\tilde{\theta}_{s}\right] \circ \Phi_{\mathfrak{p}}\left(v^{i} M_{x}\right)=\left[\tilde{\theta}_{s}\right]\left(\left[\tilde{\Delta}\left(w_{0}^{\mathfrak{p}} x\right)\langle i\rangle\right]\right)=\left[\tilde{\Delta}\left(w_{0}^{\mathfrak{p}} x\right)\langle i+1\rangle\right]+\left[\tilde{\Delta}\left(w_{0}^{\mathfrak{p}} x s\right)\langle i\rangle\right]$ by Proposition 7.3. On the other hand, $\Phi_{\mathfrak{p}}\left(v^{i} M_{x} C_{s}\right)=\Phi_{\mathfrak{p}}\left(v^{i} M_{x s}+v^{i+1} M_{x}\right)=\left[\tilde{\Delta}\left(w_{0}^{\mathfrak{p}} x s\right)\langle i\rangle\right]+$ $\left[\tilde{\Delta}\left(w_{o}^{\mathfrak{p}} x\right)\langle i+1\rangle\right]$ by Lemma 7.5

Now let $x s \in W^{\mathfrak{p}}$ and $x s<x$ (hence also $\left.w_{0}^{\mathfrak{p}} x s<w_{0}^{\mathfrak{p}} x\right)$. Then $\left[\tilde{\theta}_{s}\right] \circ \Phi_{\mathfrak{p}}\left(v^{i} M_{x}\right)=$ $\left[\tilde{\theta}_{s}\right]\left(\left[\tilde{\Delta}\left(w_{0}^{\mathfrak{p}} x\right)\langle i\rangle\right]\right)=\left[\tilde{\Delta}\left(w_{0}^{\mathfrak{p}} x s\right)\langle i\rangle\right]+\left[\tilde{\Delta}\left(w_{0}^{\mathfrak{p}} x s\right)\langle i-1\rangle\right]=\Phi_{\mathfrak{p}}\left(v^{i} M_{x}+v^{i-1} M_{x s}\right)=$ $\Phi_{\mathfrak{p}}\left(v^{i} M_{x} C_{s}\right)$ by Proposition 7.3 and Lemma 7.5

If $x s \notin W^{\mathfrak{p}}$, then $\left[\tilde{\theta}_{s}\right] \circ \Phi_{\mathfrak{p}}\left(v^{i} M_{x}\right)=\left[\tilde{\theta}_{s}\right]\left(\left[\tilde{\Delta}\left(w_{0}^{\mathfrak{p}} x\right)\langle i\rangle\right]\right)=\left[\tilde{\Delta}\left(w_{0}^{\mathfrak{p}} x\right)\langle i+1\rangle\right]+$ $\left[\tilde{\Delta}\left(w_{0}^{\mathfrak{p}} x\right)\langle i-1\rangle\right]=\Phi_{\mathfrak{p}}\left(v^{i+1} M_{x}+v^{i-1} M_{x s}\right)=\Phi_{\mathfrak{p}}\left(v^{i} M_{x} C_{s}\right)$ by Proposition 7.3 and Lemma 7.5

Remark 7.8. By a categorification of $\mathcal{M}_{\mathfrak{p}}$ we understand an abelian category $\mathcal{A}$ together with an exact auto-functor $G$ and exact endofunctors $F_{s}$ for any $s \in S$ such that the following hold:

(C1) $[\mathcal{A}]$ becomes a $\mathbb{Z}\left[v, v^{-1}\right]$-module via $v^{i} \cdot[M]=\left[G^{i} M\right]$ for any $i \in \mathbb{Z}$ and there is an isomorphism $\Phi_{\mathfrak{p}}: \mathcal{M}_{\mathfrak{p}} \cong[\mathcal{A}]$ of $\mathbb{Z}\left[v, v^{-1}\right]$-modules.

(C2) For all $s \in S$ we have an isomorphism of functors $G F_{s} \cong F_{s} G$. 
(C3) $\left[F_{s}\right]$ and $[G]$ satisfy the following relations (compare Lemma 7.5 ) for any $x \in W^{\mathfrak{p}}$ :

$$
\left[F_{s}\right]\left(\Phi_{\mathfrak{p}}\left(H_{x}\right)\right)= \begin{cases}\Phi_{\mathfrak{p}}\left(H_{x s}\right)+[G]\left(\Phi_{\mathfrak{p}}\left(H_{x}\right)\right) & \text { if } x s \in W^{\mathfrak{p}}, x s>x, \\ \Phi_{\mathfrak{p}}\left(H_{x s}\right)+\left[G^{-1}\right]\left(\Phi_{\mathfrak{p}}\left(H_{x}\right)\right) & \text { if } x s \in W^{\mathfrak{p}}, x s<x, \\ {[G]\left(\Phi_{\mathfrak{p}}\left(H_{x}\right)\right)+\left[G^{-1}\right]\left(\Phi_{\mathfrak{p}}\left(H_{x}\right)\right)} & \text { if } x s \notin W^{\mathfrak{p}} .\end{cases}
$$

In this sense, gmof $-B$ is a categorification of $\mathcal{M}_{\mathfrak{p}}$ with $F_{s}=\tilde{\theta}_{s}$ and $G=\langle 1\rangle$, the grading shift by 1 . The results of [St3] show that gmof $-A$ can be considered as a categorification of $\mathcal{H}$. In [St2] it is proved that a graded version of the principal block of a parabolic category $\mathcal{O}$ can be considered as categorification of the Hecke module $\mathcal{N}_{\mathfrak{p}}=\mathbb{Z}\left[v, v^{-1}\right] \otimes_{\mathcal{H}_{\mathfrak{p}}} \mathcal{H}$, where $\mathbb{Z}\left[v, v^{-1}\right]$ is considered as an $\mathcal{H}_{\mathfrak{p}}$-bimodule where $H_{s}$ acts by multiplication with $-v$ (see [So1]).

Example 7.9. We consider the case $\mathfrak{g}=\mathfrak{s l}(n+1, \mathbb{C})$ with $\mathfrak{p}$ such that $\mathfrak{a} \cong \mathfrak{s l}(n, \mathbb{C})$. We have $W \cong S_{n+1}=\left\langle s_{i} \mid 1 \leq i \leq n\right\rangle$ and assume $W_{\mathfrak{p}}=\left\langle s_{j} \mid 1<j \leq n\right\rangle$. The expressions $x_{j}=s_{1} s_{2} \cdots s_{j}$ are reduced for $0 \leq j \leq n$ (here $x_{0}=e$ ), hence $W^{\mathfrak{p}}=\left\{x_{i} \mid 0 \leq i \leq n\right\}$. Let $M_{i}=M_{x_{i}}$ denote the standard basis of $\mathcal{M}^{\mathfrak{p}}$. As a $\mathbb{Z}-$ module $\mathcal{M}^{\mathfrak{p}}$ is isomorphic to $\mathbb{Z}\left[v, v^{-1}\right] \otimes_{\mathbb{Z}} \mathbb{Z}^{n+1}$. The homomorphism $\left[\tilde{\theta}_{s_{j}}\right]-[\operatorname{ID}\langle 1\rangle]$ on $[\mathrm{gmof}-B]$ is then described by the action of the Hecke algebra as follows:

$$
M_{i} H_{s_{j}}= \begin{cases}M_{i+1} & \text { if } i=j-1, \\ M_{i-1}+\left(v^{-1}-v\right) M_{i} & \text { if } i=j, \\ v^{-1} M_{i} & \text { otherwise. }\end{cases}
$$

This provides a representation of the Braid group, which is not isomorphic to the Bureau representation.

\section{The graded Properly Stratified structure on $\mathcal{O}_{0}^{\mathfrak{p}-\text { pres }}$}

In this section we show that the properly stratified structure on $\mathcal{O}_{0}^{\mathfrak{p}-\text { pres }}$ admits a graded lift. The first natural question is whether the simple objects in $\mathcal{O}_{0}^{\mathfrak{p}-\text { pres }}$ have graded lifts:

Lemma 8.1. For any $w \in W(\mathfrak{p})$, the module $S(w)$ is gradable. A lift is unique up to isomorphism and grading shift.

Proof. Recall that $S(w) \cong P(w) / \operatorname{Tr}_{P_{\mathfrak{p}}}(\operatorname{rad} P(w))$. Since the canonical surjection $c_{w}: \tilde{P}(w) \rightarrow \tilde{L}(w)$ is gradable, its kernel $\operatorname{rad} P(w)$ is gradable as well with a lift $\widetilde{\operatorname{rad}} P(w)$. For $P_{\mathfrak{p}}=\bigoplus_{x \in W(\mathfrak{p})} \tilde{P}(x)$ we consider now the following isomorphisms of vector spaces:

$$
\begin{aligned}
\operatorname{Hom}_{\mathfrak{g}}\left(P_{\mathfrak{p}}, \operatorname{rad} P(w)\right) & \cong \operatorname{Hom}_{\text {mof }-A}\left(\operatorname{Hom}_{\mathfrak{g}}\left(P, P_{\mathfrak{p}}\right), \operatorname{Hom}_{\mathfrak{g}}(P, \operatorname{rad} P(w))\right) \\
& \cong \bigoplus_{i \in \mathbb{Z}} \operatorname{Hom}_{\mathrm{gmof}-A}\left(\tilde{P}_{\mathfrak{p}}\langle i\rangle, \widetilde{\operatorname{rad} P}(w)\right)
\end{aligned}
$$

That is, $\operatorname{Tr}_{P_{\mathfrak{p}}(\operatorname{rad} P(w))}$ has a graded lift, $\tilde{\operatorname{Tr}}_{P_{\mathfrak{p}}}(\operatorname{rad} P(w))$ say, namely the (graded) submodule given by all images of graded morphisms from $\bigoplus_{i \in \mathbb{Z}} \tilde{P}_{\mathfrak{p}}\langle i\rangle$ to $P(w)$. Hence, a graded lift of $S(w)$ is given by $\tilde{P}(w) / \tilde{T}_{P_{\mathfrak{p}}}(\operatorname{rad} P(w))$. Since $S(w)$ is indecomposable by Lemma [2.7, the uniqueness of such a lift follows from Lemma 7.1 
We denote by $\tilde{S}(w)$ the (unique up to isomorphism) graded lift of $S(w)$ with head concentrated in degree zero. Note that the proof of Lemma 8.1 shows that the canonical map $\tilde{P}(w) \rightarrow \widetilde{S}(w)$ is a homomorphism of graded $A$-modules. Moreover, the modules $S(w)\langle i\rangle, w \in W(\mathfrak{p}), i \in \mathbb{Z}$, form a complete set of simple objects in gmof $-B$.

Remark 8.2. Recall that for $w \in W(\mathfrak{p})$ the proper standard module $\bar{\Delta}(w)$ is isomorphic to $\Delta(w) / \operatorname{Tr}_{\Delta(w)}(\operatorname{rad} \Delta(w))$. Hence we get the gradability of $\bar{\Delta}(w)$ by completely analogous arguments as in the proof of Lemma 8.1. Let $\tilde{\bar{\Delta}}(w)$ denote the (up to isomorphism) unique lift such that the head is concentrated in degree 0 .

We say $M \in$ gmof $-A$ has a graded Verma flag if it has a filtration with subquotients isomorphic to some $\tilde{M}(x)\langle k\rangle$ with $x \in W, j \in \mathbb{Z}$. An example for such objects is given by the following.

Proposition 8.3. Let $w \in W(\mathfrak{p})$. Then $\tilde{\Delta}(w) \in$ gmof $-A$ has a graded Verma flag. Moreover, the occurring subquotients, all with multiplicity 1 , are exactly $\tilde{M}(y w)\langle l(y)\rangle, y \in W_{\mathfrak{p}}$.

The first part will follow from the following more general lemma.

Lemma 8.4. Let $M \in$ gmof $-A$ such that $\mathrm{f}(M) \cong \operatorname{Hom}_{\mathfrak{g}}(P, \check{M})$ for some $\check{M} \in \mathcal{O}_{0}$ which has a Verma flag. Then $M$ has a graded Verma flag.

Proof. We choose a surjection of $A$-modules (i.e., not necessarily graded) $M \stackrel{f}{\rightarrow}$ $\tilde{M}(x)$ for some $x \in W$. Let $f=\sum_{i \in \mathbb{Z}} f_{i}$ be the decomposition into its graded components. Since $f$ is surjective, there is at least one $i$ such that the image of $f_{i}$ is not contained in the radical of $\tilde{M}(x)$, i.e., $f_{i}$ is surjective. The kernel, $K$ say, of $f_{i}$ is then a graded $A$-module. On the other hand, an object $N \in \mathcal{O}_{0}$ has a Verma flag if and only if $\operatorname{Ext}_{\mathcal{O}}^{i}\left(N, M(y)^{-}\right)=0$ for any $y \in W$. Hence, $K$ satisfies again the assumptions and the existence of a graded Verma flag follows inductively.

Proof of Proposition 8.3. It remains to prove the multiplicity formulae. Let us first consider the case where $w \in W(p)$ is minimal, i.e., $\tilde{\Delta}\left(w_{0}^{\mathfrak{p}}\right)=\tilde{P}\left(w_{0}^{\mathfrak{p}}\right)$. By [BGS, 3.11] or [St3, 8.1], the number how often $\tilde{M}(y)\langle i\rangle$ occurs in a graded Verma flag is equal to the coefficient of $v^{i}$ in the Kazhdan-Lusztig polynomial $h_{y, w_{0}^{\mathrm{p}}}$ (in the notation of [So1]). This number is therefore nonzero (and moreover equal to 1) if and only if $i=l(y)$ and $y \in W_{\mathfrak{p}}$ by [So1, Proposition 2.9]. This gives the starting point for our induction. So, let us assume the formula to be true for $w \in W(\mathfrak{p})$. If $w$ is not maximal we choose a simple reflection $s$ such that $w s>w$ and $w s \in W(\mathfrak{p})$. Then (with [Hu, 1.10]) yws $>y w$ for any $y \in W_{\mathfrak{p}}$. The exact sequences of Proposition 7.3 and (7.1) imply therefore by induction hypotheses the following equalities in the Grothendieck group of gmof $-A$ :

$$
\begin{aligned}
{[\tilde{\Delta}(w s)] } & =\left[\tilde{\theta}_{s} \tilde{\Delta}(w)\right]-[\tilde{\Delta}(w)\langle 1\rangle] \\
& =\sum_{y \in W(\mathfrak{p})}\left(\left[\tilde{\theta}_{s} \tilde{M}(y w)\langle l(y)\rangle\right]-[\tilde{M}(y w)\langle l(y)+1\rangle]\right) \\
& =\sum_{y \in W(\mathfrak{p})}([\tilde{M}(y w)\langle l(y)+1\rangle]+[\tilde{M}(y w s)\langle l(y)\rangle]-[\tilde{M}(y w)\langle l(y)+1\rangle]) \\
& =\sum_{y \in W(\mathfrak{p})}[\tilde{M}(y w s)\langle l(y)\rangle] .
\end{aligned}
$$


This gives the required multiplicity formulae.

The following proposition is a graded analogue of property (PS1):

Proposition 8.5. Let $w \in W(\mathfrak{p})$. The graded module $\tilde{P}(w) \in$ gmof $-B$ has a $\tilde{\Delta}$ flag, i.e., a filtration with subquotients isomorphic to some $\tilde{\Delta}(x)\langle j\rangle$, where $x \in W(\mathfrak{p})$ and $j \in \mathbb{Z}$.

Proof. We prove again a more general fact (note that it directly generalizes to gmof $-A)$ :

Lemma 8.6. Let $M \in$ gmof $-B$ such that $\mathrm{f}(M) \cong \operatorname{Hom}_{\mathfrak{g}}\left(P_{\mathfrak{p}}, \check{M}\right)$ for some $\check{M} \in$ $\mathcal{O}_{0}^{\mathfrak{p}-\text { pres }}$ which has a standard flag. Then $M$ has a $\tilde{\Delta}$-flag.

We choose a surjection of (not necessarily graded) $B$-modules $M \stackrel{f}{\rightarrow} \tilde{\Delta}(x)$ for some $x \in W$. Let $f=\sum_{i \in \mathbb{Z}} f_{i}$ be the decomposition into its graded components. Since $f$ is surjective, there is at least one $i$ such that the image of $f_{i}$ is not contained in the radical of $\tilde{\Delta}(x)$, i.e., $f_{i}$ is surjective. The kernel, $K$ say, of $f_{i}$ is then a graded $B$-module. Since $\check{M} \in \mathcal{O}_{0}^{\mathfrak{p}-\text { pres }}$ has a standard flag and surjects onto $\Delta(x)$, we have $\mathrm{f} K \cong \operatorname{Hom}_{\mathfrak{g}}\left(P_{\mathfrak{p}}, N\right)$ for some $N \in \mathcal{O}_{0}^{\mathfrak{p}-\text { pres }}$. On the other hand, an object $N \in \mathcal{O}_{0}^{\mathfrak{p}-\text { pres }}$ has a standard flag if and only if $\operatorname{Ext}_{\mathcal{O}_{0}^{\mathfrak{p}-\text { pres }}}^{i}(N, \bar{\nabla}(y))=0$ for any $y \in W(\mathfrak{p})$ (see [D1, Theorem 5]). Since also $\operatorname{Ext}_{\mathcal{O}_{0}^{\mathfrak{p}-p r e s}}^{2}(\Delta(x), \bar{\nabla}(y))=0$ for $x$, $y \in W(\mathfrak{p})$ (again by [D] Theorem 5]), $K$ satisfies again the assumptions and the existence of a graded Verma flag follows inductively.

We call a finite-dimensional associative graded algebra $D$ with a fixed order $\leq$ on the set $I$ of isomorphism classes of simple graded $D$-modules (the latter will be denoted by $S(i), i \in I)$ graded properly stratified provided that there exist graded $D$-modules $\Delta(i)$ and $\bar{\Delta}(i), i \in I$, such that the graded versions of (PS1), (PS2) and (PS3) hold. We summarize the results of this section in the following.

Proposition 8.7. Set $\tilde{S}(w)\langle j\rangle>\tilde{S}\left(w^{\prime}\right)\left\langle j^{\prime}\right\rangle$ if $w>w^{\prime}$ or $w=w^{\prime}$ and $j>j^{\prime}$. Then the algebra $B$ is graded properly stratified. The modules $\tilde{\Delta}(w)$ and $\tilde{\bar{\Delta}}(w)$, where $w \in W(\mathfrak{p})$, form the sets of graded standard and proper standard modules respectively.

Proof. By Proposition 8.5, all projectives have a $\tilde{\Delta}$-flag. By the formulae BGS 3.11] and [St3, 8.1], all projectives are positively graded having only their simple heads concentrated in degree zero. This ensures (PS1), since the additional condition on the filtration holds after forgetting the grading. With the positivity of the grading on projectives the property (PS2) follows from the definition of the graded lifts and the existence of the corresponding filtration after forgetting the grading. To see property (PS3), we take the graded Verma flag from Proposition 8.3. Since the arguments in the proof of Lemma 8.1 show that the composition of functors $\mathfrak{i j}$ induces a functor on gmof $-A$, we can apply it to the graded Verma flag. The result will be the required filtration.

Note, that the isomorphism classes of standard objects in $\mathcal{O}_{0}^{\mathfrak{p}-\text { pres }}$ do not form a basis of the corresponding Grothendieck group. The reason for this is that (with the usual ordering on the isomorphism classes of simple modules) the matrix $[\Delta(w)$ : $L(y)]$ is upper triangular, but has positive integers on the diagonal in general. In 
the graded version, we get 1's on the diagonal, hence $[\tilde{S}(w)]=\sum_{y, j} c_{y, j}[\Delta(y)\langle j\rangle]$ with unique $c_{y, j} \in \mathbb{Z}$ (but probably infinitely many nonzero coefficients!).

Corollary 8.8. Each of the following sets forms a $\mathbb{Z}$-basis of $[\mathrm{gmof}-B]$ :

$$
\begin{aligned}
& \{[\tilde{S}(w)\langle i\rangle \mid w \in W(\mathfrak{p}), i \in \mathbb{Z}]\}, \\
& \{[\tilde{\bar{\Delta}}(w)\langle i\rangle \mid w \in W(\mathfrak{p}), i \in \mathbb{Z}]\} .
\end{aligned}
$$

Proof. The first claim is obvious and the second one follows from the fact that the transformation matrix from the basis given by simple modules to the basis given by proper standard modules is triangular and $[\bar{\Delta}(w): S(w)]=1$.

Remark 8.9. Let $x, y \in W(\mathfrak{p})$. The graded duals $(\tilde{\bar{\Delta}}(x))^{\circledast}$ of $(\tilde{\bar{\Delta}}(y))$ (see [St3] define the dual proper standard modules of gmof $-B$. By general reasons (see $[\overline{D l}]$ ), we get the following multiplicity formulae:

$$
\begin{aligned}
{[\tilde{P}(x): \tilde{\Delta}(y)\langle i\rangle] } & =\left[(\tilde{\bar{\Delta}}(y))^{\circledast}\langle i\rangle: \tilde{S}(x)\right] \\
& \stackrel{(1)}{=}\left[\tilde{M}(y)^{\circledast}\langle i\rangle: \tilde{L}(x)\right] \\
& \stackrel{(2)}{=}\left[\tilde{M}(y)^{\circledast}: \tilde{L}(x)\langle-i\rangle\right] \\
& \stackrel{(3)}{=}[\tilde{M}(y): \tilde{L}(x)\langle i\rangle] \\
& \stackrel{(4)}{=}[\tilde{P}(x): \tilde{M}(y)\langle i\rangle] .
\end{aligned}
$$

The equality (1) follows from the construction of the graded proper standard flag in the proof of Proposition 8.7. The equality (2) is just a grading argument. Finally (3) and (4) are given by [St3, Section 7]. Now, the last number is given as the coefficient of $v^{i}$ in the Kazhdan-Lusztig polynomial $h_{y, x}$ (in the notation of [So1]) and this is equal to the coefficient of $v^{i}$ in $m_{w_{0}^{\mathrm{p}} y, w_{0}^{\mathrm{p}} x}$ by the formula [So1, Proposition 3.4]. This implies that, under the isomorphism of Theorem [7.7 the isomorphism class $[\tilde{P}(w)]$ corresponds to the self-dual Kazhdan-Lusztig element $\underline{M}_{w}$ in the notation of $[\mathrm{So1}]$.

\section{EXAmPLES}

9.1. $\mathcal{O}_{0}^{\mathfrak{p}-\text { pres }}$ for $\mathfrak{s l}(3, \mathbb{C})$. Let $\mathfrak{g}=\mathfrak{s l}(3, \mathbb{C})$ and $\mathfrak{p}=\mathfrak{b}$ be the standard Borel subalgebra of $\mathfrak{g}$. The Weyl group of $\mathfrak{g}$ is isomorphic to the symmetric group $S_{3}$ and is equal to $\{e, s, t, s t, t s, s t s=t s t\}$, where $s$ and $t$ are standard simple reflections. To simplify our notation, let us denote the simple modules $L(e), L(s), L(t), L(s t)$, $L(t s)$, and $L(s t s)$ by $1,2,3,4,5$, and 6 respectively. The first row of Figure 1 shows the radical filtrations of the Verma modules in the category $\mathcal{O}_{0}$. Using Verma modules, one computes the action of $\theta_{s}$ and $\theta_{t}$ on simple modules and gets the following radical filtrations for translated simple modules:

$$
\begin{aligned}
& \theta_{s}(1)=0, \quad \theta_{s}(2)=\begin{array}{c}
2 \\
14 \\
2
\end{array}, \quad \theta_{s}(3)=0, \quad \theta_{s}(4)=0, \quad \theta_{s}(5)=\begin{array}{l}
5 \\
3 \\
5
\end{array}, \quad \theta_{s}(6)=\begin{array}{l}
6 \\
4 \\
6
\end{array} ; \\
& \theta_{t}(1)=0, \quad \theta_{t}(2)=0, \quad \theta_{t}(3)=\begin{array}{c}
3 \\
15 \\
3
\end{array}, \quad \theta_{t}(4)=\begin{array}{l}
4 \\
2 \\
4
\end{array}, \quad \theta_{t}(5)=0, \quad \theta_{t}(6)=\begin{array}{l}
6 \\
5 \\
6
\end{array} \text {. }
\end{aligned}
$$




\begin{tabular}{|c|c|c|c|c|c|c|}
\hline$x \backslash y$ & $e$ & $s$ & $t$ & st & $t s$ & sts \\
\hline$e$ & $\begin{array}{c}1 \\
23 \\
45 \\
6\end{array}$ & $\begin{array}{c}2 \\
45 \\
6\end{array}$ & $\begin{array}{c}3 \\
45 \\
6\end{array}$ & $\begin{array}{l}4 \\
6\end{array}$ & $\begin{array}{l}5 \\
6\end{array}$ & 6 \\
\hline$s$ & $\begin{array}{c}2 \\
45 \\
6 \\
\end{array}$ & $\begin{array}{c}2 \\
145 \\
36\end{array}$ & $\begin{array}{l}4 \\
6\end{array}$ & $\begin{array}{c}4 \\
36 \\
5\end{array}$ & 6 & $\begin{array}{l}6 \\
5\end{array}$ \\
\hline$t$ & $\begin{array}{c}3 \\
45 \\
6\end{array}$ & $\begin{array}{l}5 \\
6\end{array}$ & $\begin{array}{c}3 \\
145 \\
26\end{array}$ & 6 & $\begin{array}{c}5 \\
26 \\
4\end{array}$ & $\begin{array}{l}6 \\
4\end{array}$ \\
\hline st & $\begin{array}{l}4 \\
6\end{array}$ & 6 & $\begin{array}{c}4 \\
26 \\
5\end{array}$ & $\begin{array}{l}6 \\
5\end{array}$ & $\begin{array}{c}62 \\
145 \\
3\end{array}$ & $\begin{array}{c}6 \\
54 \\
3\end{array}$ \\
\hline$t s$ & $\begin{array}{l}5 \\
6\end{array}$ & $\begin{array}{c}5 \\
36 \\
4\end{array}$ & 6 & $\begin{array}{c}36 \\
154 \\
2\end{array}$ & $\begin{array}{l}6 \\
4\end{array}$ & $\begin{array}{c}6 \\
54 \\
2\end{array}$ \\
\hline sts & 6 & $\begin{array}{l}6 \\
4\end{array}$ & $\begin{array}{l}6 \\
5\end{array}$ & $\begin{array}{c}6 \\
45 \\
2\end{array}$ & $\begin{array}{c}6 \\
45 \\
3\end{array}$ & $\begin{array}{c}6 \\
45 \\
23 \\
1\end{array}$ \\
\hline
\end{tabular}

Figure 1. The shuffled Verma modules $M(x, y) \in \mathcal{O}_{0}$

Under the categorification morphism, the functors $\theta_{s}$ and $\theta_{t}$ are represented by the following matrices, given in the basis of standard modules:

$$
\left[\tilde{\theta}_{s}\right]=\left(\begin{array}{cccccc}
v & 1 & 0 & 0 & 0 & 0 \\
1 & v^{-1} & 0 & 0 & 0 & 0 \\
0 & 0 & v & 0 & 1 & 0 \\
0 & 0 & 0 & v & 0 & v \\
0 & 0 & 1 & 0 & v^{-1} & 0 \\
0 & 0 & 0 & 1 & 0 & v^{-1}
\end{array}\right), \quad\left[\tilde{\theta}_{t}\right]=\left(\begin{array}{cccccc}
v & 0 & 1 & 0 & 0 & 0 \\
0 & v & 0 & 1 & 0 & 0 \\
1 & 0 & v^{-1} & 0 & 0 & 0 \\
0 & 1 & 0 & v^{-1} & 0 & 0 \\
0 & 0 & 0 & 0 & v & 1 \\
0 & 0 & 0 & 0 & 1 & v^{-1}
\end{array}\right) .
$$

Using the action of $\theta_{s}$ and $\theta_{t}$ on simple modules and the definition of $C_{s}$ and $C_{t}$ it is possible to compute all shuffled Verma modules $M(x, y), x, y \in W$. Their radical filtrations are collected in Figure 1.

9.2. $\mathcal{O}_{0}^{\mathfrak{p}-\text { pres }}$ for a proper parabolic in $\mathfrak{s l}(3, \mathbb{C})$. Now let us assume that $\mathfrak{g}=$ $\mathfrak{s l}(3, \mathbb{C})$ and $\mathfrak{p}$ is a parabolic subalgebra of $\mathfrak{g}$, such that $W_{\mathfrak{p}}=\{e, s\}$. Then $W(\mathfrak{p})=$ $\{s, s t, s t s\}$. Using the above tables and the definition of $C_{s}$ and $C_{t}$ one computes all shuffled standard modules $\tilde{\Delta}(x, y), x \in W(\mathfrak{p}), y \in W$. Their graded filtrations are collected in Figure 2 and Figure 3. The first columns describe the graded lifts of the usual standard modules.

In the basis of standard modules the matrices of the categorification morphism are the following:

$$
\left[\tilde{\theta}_{s}\right]=\left(\begin{array}{ccc}
v+v^{-1} & 0 & 0 \\
0 & v & 1 \\
0 & 1 & v^{-1}
\end{array}\right), \quad\left[\tilde{\theta}_{t}\right]=\left(\begin{array}{ccc}
v & 1 & 0 \\
1 & v^{-1} & 0 \\
0 & 0 & v+v^{-1}
\end{array}\right) .
$$




\begin{tabular}{|c|c|c|c|c|c|c|}
\hline$x \backslash y$ & $e$ & $s$ & $t$ & $s t$ & $t s$ & sts \\
\hline$s$ & $\begin{array}{c}2 \\
451 \\
623 \\
45 \\
6\end{array}$ & $\begin{array}{c}2 \\
451 \\
623 \\
45 \\
6\end{array}$ & $\begin{array}{c}4 \\
63 \\
54 \\
6\end{array}$ & $\begin{array}{c}4 \\
36 \\
54 \\
6\end{array}$ & $\begin{array}{l}6 \\
5 \\
6\end{array}$ & $\begin{array}{l}6 \\
5 \\
6\end{array}$ \\
\hline$s t$ & $\begin{array}{c}4 \\
36 \\
54 \\
6\end{array}$ & $\begin{array}{l}6 \\
5 \\
6\end{array}$ & $\begin{array}{c}4 \\
236 \\
5154 \\
26\end{array}$ & $\begin{array}{l}6 \\
5 \\
6\end{array}$ & $\begin{array}{c}62 \\
1455 \\
362 \\
4\end{array}$ & $\begin{array}{c}6 \\
54 \\
36 \\
4\end{array}$ \\
\hline sts & $\begin{array}{l}6 \\
5 \\
6\end{array}$ & $\begin{array}{c}6 \\
45 \\
36 \\
4\end{array}$ & $\begin{array}{l}6 \\
5 \\
6\end{array}$ & $\begin{array}{c}6 \\
45 \\
236 \\
154 \\
2\end{array}$ & $\begin{array}{c}6 \\
54 \\
36 \\
4\end{array}$ & $\begin{array}{c}6 \\
45 \\
236 \\
154 \\
2\end{array}$ \\
\hline
\end{tabular}

Figure 2. The shuffled standard objects $\tilde{\Delta}(x, y)$ as objects in gmof $-A$

\begin{tabular}{|c|c|c|c|c|c|c|}
\hline$x \backslash y$ & $e$ & $s$ & $t$ & $s t$ & $t s$ & sts \\
\hline \multirow{5}{*}{$s$} & 2 & $\overline{2}$ & & & \multirow{5}{*}{$\begin{array}{l}6 \\
6\end{array}$} & \multirow{5}{*}{$\begin{array}{l}6 \\
6\end{array}$} \\
\hline & 4 & 4 & $\begin{array}{l}4 \\
6\end{array}$ & $\begin{array}{l}4 \\
6\end{array}$ & & \\
\hline & 62 & 62 & 0 & 4 & & \\
\hline & 4 & 4 & 4 & 4 & & \\
\hline & 6 & 6 & & & & \\
\hline \multirow{4}{*}{$s t$} & 4 & & 4 & & 62 & 6 \\
\hline & 6 & 6 & 26 & 6 & 4 & 4 \\
\hline & 4 & 6 & 4 & 6 & 62 & 6 \\
\hline & 6 & & 26 & & 4 & 4 \\
\hline \multirow{4}{*}{ sts } & \multirow{4}{*}{$\begin{array}{l}6 \\
6\end{array}$} & 6 & \multirow{4}{*}{$\begin{array}{l}6 \\
6\end{array}$} & 6 & 6 & 6 \\
\hline & & 4 & & 4 & 4 & $\begin{array}{c}4 \\
2\end{array}$ \\
\hline & & 6 & & 26 & 6 & 26 \\
\hline & & 4 & & $\begin{array}{l}4 \\
2\end{array}$ & 4 & $\begin{array}{l}4 \\
2\end{array}$ \\
\hline
\end{tabular}

Figure 3 . The shuffled standard objects $\tilde{\Delta}(x, y)$ as objects in gmof $-B$

\section{ACKNOWLEDGMEnts}

A part of this research was done during the visit of the first author to Leicester, which was supported by EPSRC. An essential part of the paper was written during the visit of the second author to Uppsala University, which was supported by The Royal Swedish Academy of Sciences. The financial support of the Academy and the hospitality of Uppsala University are gratefully acknowledged. The authors thank Steffen König for many stimulating discussions, in particular, for the information about the paper Ric. We thank Henning Haahr Andersen for comments on the 
preliminary version. We also thank the referee for pointing out the connection with the thick category $\mathcal{O}$.

\section{REFERENCES}

[AJS] H. H. Andersen, J. C. Jantzen, W. Soergel, Representations of quantum groups at a pth root of unity and of semisimple groups in characteristic $p$ : independence of $p$. Astérisque No. 220 (1994). MR95j:20036

[AL] H. H. Andersen, N. Lauritzen, Twisted Verma modules, in: Studies in Memory of Issai Schur, 1-26, v. 210, Progress in Math., Birkhäuser, Basel, 2002. MR2004d:17005

[Au] M. Auslander, Representation theory of Artin algebras I. Commun. in Alg. 1 (1974), 177-268. MR50:2240

[Ba] E. Backelin, The Hom-spaces between projective functors. Represent. Theory 5 (2001), 267-283 (electronic). MR2002f:17007

[Be] I. N. Bernstein, Trace in categories. Operator algebras, unitary representations, enveloping algebras, and invariant theory (Paris, 1989), 417-423, Progr. Math., 92, Birkhäuser Boston, Boston, MA, 1990. MR 92d:17010

[BFK] I. N. Bernstein, I. Frenkel, M. Khovanov, A categorification of the Temperley-Lieb algebra and Schur quotients of $U\left(\mathfrak{s l}_{2}\right)$ via projective and Zuckerman functors, Selecta Math. (N.S.), 5 (1999), no. 2, 199-241. MR2000i:17009

[BG] I. N. Bernstein, S. I. Gelfand, Tensor products of finite- and infinite-dimensional representations of semisimple Lie algebras. Compositio Math. 41 (1980), no. 2, 245-285. MR 82c:17003

[BGG] I. N. Bernstein, I. M. Gelfand, S. I. Gelfand, A certain category of $\mathfrak{g}$-modules. Funkcional. Anal. i Prilozen. 10 (1976), no. 2, 1-8. MF 53:10880

[BGS] A. Beilinson, V. Ginzburg, W. Soergel, Koszul duality patterns in representation theory. J. Amer. Math. Soc. 9 (1996), no. 2, 473-527. MR.96k:17010

[Bo] N. Bourbaki, Lie groups and Lie algebras. Chapters 4-6. Elements of Mathematics. Springer-Verlag, Berlin, 2002. MR2003a:17001

[CPS] E. Cline, B. Parshall, L. Scott, Finite-dimensional algebras and highest weight categories. J. Reine Angew. Math. 391 (1988), 85-99. MR.90d:18005

[CI] D. Collingwood, R. S. Irving, A decomposition theorem for certain self-dual modules in the category $\mathcal{O}$. Duke Math. J. 58 (1989), no. 1, 89-102. MR90k:17010

[De] V. Deodhar, On some geometric aspects of Bruhat orderings. II. The parabolic analogue of Kazhdan-Lusztig polynomials. J. Algebra 111 (1987), no. 2, 483-506. MR89a:20054

[Di] J. Dixmier, Enveloping algebras. Graduate Studies in Mathematics, 11. American Mathematical Society, Providence, RI, 1996. MR97c:17010

[Dl] V. Dlab, Properly stratified algebras. C. R. Acad. Sci. Paris Sér. I Math. 331 (2000), no. 3, 191-196. MR2001e:16016

[DFO] Yu. Drozd, S. Ovsienko, V. Futorny, The Harish-Chandra S-homomorphism and $\mathfrak{G}$ modules generated by a semiprimitive element. Ukrain. Mat. Zh. 42 (1990), no. 8, 10311037; translation in Ukrainian Math. J. 42 (1990), no. 8, 919-924 (1991) MR92d:17003

[En] T. Enright, On the fundamental series of a real semisimple Lie algebra: their irreducibility, resolutions and multiplicity formulae. Ann. of Math. (2) 110 (1979), no. 1, 1-82. MR81a:17003

[FKM1] V. Futorny, S. König, V. Mazorchuk, Categories of induced modules and standardly stratified algebras. Algebr. Represent. Theory 5 (2002), no. 3, 259-276. MR2003g:17005

[FKM2] V. Futorny, S. König, V. Mazorchuk, S-subcategories in $\mathcal{O}$. Manuscripta Math. 102 (2000), no. 4, 487-503. MR2001h:17022

[GJ] O. Gabber, A. Joseph, Towards the Kazhdan-Lusztig conjecture. Ann. Sci. École Norm. Sup. (4) 14 (1981), no. 3, 261-302. MR83e:17009

[Hu] J. Humphreys, Reflection groups and Coxeter groups. Cambridge Studies in Advanced Mathematics, 29. Cambridge University Press, Cambridge, 1990. MR.92h:20002

[Ir1] R. Irving, Shuffled Verma modules and principal series modules over complex semisimple Lie algebras. J. London Math. Soc. (2) 48 (1993), no. 2, 263-277. MR94i:17013

[Ir2] R. Irving, Projective modules in the category $\mathcal{O}_{S}$ : self-duality. Trans. Amer. Math. Soc. 291 (1985), no. 2, 701-732. MR.87i:17005 
[Ja] J. C. Jantzen, Einhüllende Algebren halbeinfacher Lie-Algebren. Ergebnisse der Mathematik und ihrer Grenzgebiete 3. Springer-Verlag, Berlin, 1983. MR86c:17011

$[\mathrm{Kh}] \quad$ O. Khomenko, Categories with projective functors, $\mathrm{PhD}$ thesis. Universität Freiburg (Germany), 2004.

[KL] D. Kazhdan, G. Lusztig, Representations of Coxeter groups and Hecke algebras. Invent. Math. 53 (1979), no. 2, 165-184. MR81j:20066

[KM1] S. König, V. Mazorchuk, Enright's completions and injectively copresented modules. Trans. Amer. Math. Soc. 354 (2002), no. 7, 2725-2743. MR2003c:17009

[KM2] S. König, V. Mazorchuk, An equivalence of two categories of $\mathrm{sl}(n, \mathbb{C})$-modules. Algebr. Represent. Theory 5 (2002), no. 3, 319-329. MR2003i:17009

[Ma] V. Mazorchuk, Twisted and shuffled filtrations on tilting modules. Math. Reports of the Academy of Science of the Royal Society of Canada 25 (2003), no. 1, 26-32. MR2004c:17013

[Rin] C. M. Ringel, The category of modules with good filtrations over a quasi-hereditary algebra has almost split sequences. Math. Z. 208 (1991), no. 2, 209-223. MR.93c:16010

[Ric] J. Rickard, Translation functors and equivalences of derived categories for blocks of algebraic groups. Finite-dimensional algebras and related topics (Ottawa, ON, 1992), 255264, NATO Adv. Sci. Inst. Ser. C Math. Phys. Sci., 424, Kluwer Acad. Publ., Dordrecht, 1994. MR95k:20068

[So1] W. Soergel, Kazhdan-Lusztig polynomials and combinatorics for tilting modules. Represent. Theory 1 (1997), 83-114 (electronic). MR.98d:17026

[So2] W. Soergel, Kategorie $\mathcal{O}$, perverse Garben und Moduln über den Koinvarianten zur Weylgruppe. J. Amer. Math. Soc. 3 (1990), no. 2, 421-445. MR91e:17007

[So3] W. Soergel, Équivalences de certaines catégories de $\mathfrak{g}$-modules. C. R. Acad. Sci. Paris Sér. I Math. 303 (1986), no. 15, 725-728. MF 88c:17011

[St1] C. Stroppel, A generalization of Joseph's functor and shuffled Harish-Chandra bimodules. in preparation.

[St2] C. Stroppel, Categorification of the Temperley-Lieb category, Tangles and cobordisms via projective functors, Preprint Aarhus University (2003).

[St3] C. Stroppel, Category $\mathcal{O}$ : Gradings and Translation Functors. J. Algebra 268 (2003), no. 1, 301-326. MF 2005290(2004i:17007)

Department of Mathematics, Uppsala University, Box 480, 751 06, Uppsala, Sweden

E-mail address: mazor@math.uu.se

Department of Mathematics, Aarhus University, Ny Munkegade 530, 8000 Aarhus C, DENMARK

E-mail address: stroppel@imf.au.dk

Current address: Department of Mathematics, University of Glasgow, 15 University Gardens, Glasgow, G12 8QW United Kingdom

E-mail address: cs@maths.gla.ac.uk 